\title{
Article
}

\section{Dynamics and Control of Typical Orbits around Saturn}

\author{
Chaojin Zhan ${ }^{1}$, Yu Jiang ${ }^{1, *}$, Hengnian Li $^{1,2}$ (D) and Yongjie Liu ${ }^{1}$ \\ 1 State Key Laboratory of Astronautic Dynamics, Xi'an Satellite Control Center, Xi'an 710043, China; \\ cjzhan_xian@163.com (C.Z.); henry_xscc@mail.xjtu.edu.cn (H.L.); liuyongjie_xian@163.com (Y.L.) \\ 2 School of Electronic and Information Engineering, Xi' an Jiaotong University, Xi'an 710049, China \\ * Correspondence: jiangyu_xian_china@163.com
}

Citation: Zhan, C.; Jiang, Y.; Li, H.; Liu, Y. Dynamics and Control of Typical Orbits around Saturn. Appl. Sci. 2022, 12, 1462. https://doi.org/ 10.3390/app12031462

Academic Editors: Jérôme Morio, Mathieu Balesdent and Loïc Brevault

Received: 22 December 2021

Accepted: 25 January 2022

Published: 29 January 2022

Publisher's Note: MDPI stays neutral with regard to jurisdictional claims in published maps and institutional affiliations.

Copyright: (c) 2022 by the authors. Licensee MDPI, Basel, Switzerland. This article is an open access article distributed under the terms and conditions of the Creative Commons Attribution (CC BY) license (https:// creativecommons.org/licenses/by/ $4.0 /)$.

\begin{abstract}
This paper investigates the dynamics of some typical orbits around Saturn, including sun-synchronous orbits, repeating ground track orbits, frozen orbits, and stationary orbits, and corresponding control methods mainly based on the mean element theory. The leading terms of Saturn's aspheric gravitational field, $J_{2}$ and $J_{4}$ terms, are used when designing the orbits around Saturn. Two control methods of sun-synchronous orbits, including initial inclination-biased method and periodic inclination-biased method, are used to damp the local time drift at the descending node, which is caused by solar gravitation and atmospheric drag. The compensation of semimajor axis and maneuver period to maintain the recursive feature of repeating ground orbits are calculated. While only $J_{2}$ and $J_{3}$ terms are taken into account, we examine the argument that the perigee of frozen orbits around Saturn should be $270 \mathrm{deg}$ to promise meaningful eccentricity. The perturbations of inclination and eccentricity of stationary orbits due to solar gravitation and solar radiation pressure are presented. Meanwhile, the preliminary control strategies of inclination perturbation and eccentricity perturbation are naturally introduced.
\end{abstract}

Keywords: Saturn; mean element theory; orbital dynamics; orbital maintenance

\section{Introduction}

Interplanetary exploration is one of the most important methods to seek the origin and evolution of the universe, suitable living planets for human beings, and the existence of other intelligent life. Over the last few decades, with the development of science and technology, we have launched numerous spaceships to explore the planets of our solar system.

Saturn, since its discovery, is absolutely one of the most attractive planets in the solar system. The tremendous thin ring consisting of many ringlets has become the famous characteristic of Saturn. Saturn is the second biggest planet in the solar system with a volume about 755 times larger than Earth. It is made predominantly of hydrogen and helium, which causes its density to be smaller than water. Saturn rotates so fast that its rotation period is only $10.656 \mathrm{~h} \mathrm{[1].} \mathrm{However,} \mathrm{its} \mathrm{orbital} \mathrm{period} \mathrm{is} \mathrm{tremendously} \mathrm{longer} \mathrm{than}$ its rotation period, at 29.4 Earth years [1]. The gravitational field of the Saturn system is extremely complicated, as Saturn is the planet with the largest amount of moons in the solar system. There are 53 known moons and 29 moons waiting to be formally confirmed [2]. The largest moon in the Saturn system, named Titan, was found to have a nitrogen-rich atmosphere similar to that of ancient Earth.

Therefore, the exploration of the Saturnian system could promote the process of searching for habitable planets and researching the evolution of planets in the solar system.

However, few spacecraft had investigated Saturn. Pioneer11 is the first spacecraft to reach the Saturn system. It reached Saturn in 1979, and discovered a narrow ring, which is named the F-ring, outside the A-ring for the first time [3]. It also first discovered the existence of the magnetosphere of Saturn. After two years, Voyager 2, the third spacecraft to visit Saturn, gained a glimpse of Saturn on its way to Uranus. Using photopolarimeter, 
Voyager 2 provided more detail about the ringlets and shepherding moons around the F-ring [4]. The most famous and longest exploration around Saturn is the Cassini-Huygens mission. Cassini-Huygens was the first mission to orbit Saturn [5]. It completed 294 orbits and sent total 453,048 images back to Earth in 13 years of discovery. These missions provided a precious opportunity for planetary scientists to observe Saturn closely.

Jacobson [6] and Jacobson et al. [7] presented Saturnian gravitational zonal harmonic coefficients using spacecraft tracking data. Waite et al. [8] observed the infalling material flux, which was calculated to be between 4800 and $45,000 \mathrm{~kg} / \mathrm{s}$, could affect the carbon content of Saturnian ionosphere and atmosphere. Müller-Wodarg et al. [9] emphasized the importance of thermospheric temperatures when examining the state of Saturnian magnetosphere.

The formation and evolution of the Saturnian interior structure [10], the magnetosphere [11], rings [12], and moons [13] around Saturn are always at the centre of the research [14]. Nevertheless, few researchers study the dynamics around Saturn.

On the contrary, the studies of dynamics around terrestrial planets had already enriched the theories of Kozai [15] and Brouwer [16]. The applications of these theories had been widely used when scientists and engineers analyzed the dynamics of artificial satellites around Earth. The design of frozen orbits was first proposed by Cutting et al. [17]. The existence of four equilibrium points of geostationary orbits was shown by Musen et al. [18]. Moreover, the periodic orbits around these equilibrium points are investigated by Lara et al. [19]. Lei [20] considered secular perturbations, combining Earth's aspheric gravitation with the luni-solar gravitation to analyze the dynamics of the medium Earth orbit navigation satellites. At the same time, some feasible research aimed to offset the effect caused by perturbations. Nazarenko [21] studied predicting the local time of the ascending node of sun-synchronous orbits around Earth. Liao et al. [22] studied a semi-analytical acquisition algorithm for repeating ground track orbits' maintenance.

Studies analyzing the dynamics around other terrestrial planets in the solar system had developed rapidly as well. Liu et al. [23] analyzed five typical orbits around Mars by using the zonal harmonic coefficients $J_{2}, J_{4}$ and a tesseral harmonic coefficient $J_{22}$. In addition, Liu et al. [24] further studied the recursive orbits around stationary points of Martian gravitational field. Ortore et al. [25] designed recursive sun-synchronous orbits of $J_{2}$ predominant planets, including Mars. Ma et al. [26] and Ma et al. [27] studied distant quasi-periodic orbits and artificial frozen orbits around Mercury.

Actually, these dynamic models are not suitable for Saturn, as Saturn is not a $J_{2}$ predominant planet. At the same time, the tesseral harmonic coefficient of Saturn $J_{22} \approx 0$. Though this research on terrestrial planets is inspiring, the more relevant studies are the dynamic analysis around gaseous planets. Liu et al. [28] analyzed the dynamics of several typical orbits around Jupiter. Jiang et al. [29] studied the initial inclination prebiased method and semimajor axis compensation strategy for sun-synchronous repeating ground track orbits around Jupiter. Jiang et al. [29]'s control strategy only considered $J_{2}$ term, while Jupiter is not a $J_{2}$ predominant planet. Though his control strategy is instructive, we still insist that the combination of $J_{2}$ and $J_{4}$ is necessary for gaseous planets, especially for non predominant $J_{2}$ celestial bodies.

In this paper, we analyze the dynamics of some typical orbits around Saturn using the aspheric gravitational model considering $J_{2}$ and $J_{4}$ terms. Then we design the sunsynchronous orbits, repeating ground track orbits, frozen orbits, and stationary orbits around Saturn based on the mean element theory $[15,16]$. While analyzing the perturbations, we divide them into three types of terms: secular terms, long-period terms, and short-period terms $[15,16]$. After that, we mainly consider secular perturbations caused by atmospheric drag, solar gravitation, and solar radiation pressure. As for sun-synchronous orbits, we first analyze the local time drift at the descending node caused by atmospheric drag and solar gravitation. Then, we take two methods, including initial inclination biased method and periodic inclination biased method, to damp the local time drift. The main perturbation we have analyzed for repeating ground track orbits is atmospheric drag. After 
we have chosen a reasonable repetition parameter, we could calculate the decay rate of orbital semimajor axis with an initial condition of semimajor axis and eccentricity. To maintain the recursive feature, we give a solution of semimajor axis compensation and control period. When we use $J_{2}$ and $J_{4}$ terms to design frozen orbits around Saturn, we naturally see a novel result that the argument of perigee of frozen orbits around Saturn must be $270^{\circ}$ to make eccentricity meaningful. At last, we give some strategies to offset the influence on inclination caused by the solar gravitation and the influence on eccentricity caused by solar radiation pressure.

In this paper, we use some traditional symbols to denote the basic parameters of Saturn and the orbital elements of spacecraft motion. $a$ is the semi-major axis of orbit. $e$ is the orbital eccentricity. $i$ is the orbital inclination. $J_{2}$ is the second-order zonal harmonic. $J_{3}$ is the third-order zonal harmonic. $J_{4}$ is the fourth-order zonal harmonic. $J_{22}$ is the second-degree and -order tesseral harmonic. $M$ is the mean anomaly. $f$ is the true anomaly. $n$ is the mean angular velocity. $n_{s}$ is the mean angular velocity of Saturn around the Sun. $p$ is the semiparameter. $R_{e}$ is the reference equatorial radius of Saturn. $r$ is the position of the spacecraft. $\Omega$ is the right ascension of the ascending node. $\lambda$ is the latitude of body-fixed coordinate system. $\mu$ is the Saturn's gravitational constant. $\varphi$ is the longitude of body-fixed coordinate system. $\omega$ is the argument of perigee. $\omega_{s}$ is the rotational angular velocity of Saturn.

In this paper, we mainly use the aspheric gravitational field zonal harmonic coefficients to design different typical orbits. Some basic parameters of Saturn are listed in Table $1[1,7,30]$. Similar to Jupiter, the magnitude of $J_{2}$ of Saturn is not predominant among the zonal harmonic coefficients $[28,29]$. Therefore, we choose the dynamic model involved $J_{2}$ and $J_{4}$ terms. The gravitational potential function could be approximated as [31]:

$$
U(r)=\frac{\mu}{r}\left[1-\frac{J_{2}}{2}\left(3 \sin ^{2} \varphi-1\right)\left(\frac{R}{r}\right)^{2}-\frac{J_{4}}{8}\left(35 \sin ^{4} \varphi-30 \sin ^{2} \varphi+3\right)\left(\frac{R}{r}\right)^{4}\right] .
$$

Table 1. Some parameters for basic model of Saturn.

\begin{tabular}{ll}
\hline$\mu\left(\mathrm{km}^{3} \mathrm{~s}^{-2}\right)$ & $37,931,207.7 \pm 1.1$ \\
Equatorial radius $R_{e}(\mathrm{~km})$ & 60,268 \\
$J_{2}\left(\times 10^{8}\right)$ & $1,629,057.33 \pm 2.8$ \\
$J_{3}\left(\times 10^{8}\right)$ & $5.89 \pm 2.3$ \\
$J_{4}\left(\times 10^{8}\right)$ & $-93,531.36 \pm 3.7$ \\
Obliquity to orbit $i_{s}$ & $26.73 \mathrm{deg}$ \\
Sidereal orbit period (days) & $10,759.22$ \\
Sidereal rotation period (hours) & 10.656 \\
\hline
\end{tabular}

\section{Sun-Synchronous Orbits and Orbital Maintenance}

The first order secular term for the precession rate of ascending node is [15,16,32]:

$$
\dot{\Omega}_{1}=-\frac{3 n J_{2} R_{e}^{2}}{2 p^{2}} \cos i \text {. }
$$

Here, the second order secular perturbation considered $J_{4}$ for the precession rate is [16]

$$
\begin{aligned}
\dot{\Omega}_{2}= & -\frac{9 n J_{2}^{2} R_{e}^{4}}{4 p^{4}}\left\{\left[\frac{3}{2}-\frac{5}{3} \sin ^{2} i-\frac{35 J_{4}}{18 J_{2}^{2}}\left(\frac{6}{7}-\frac{3}{2} \sin ^{2} i\right)\right]\right. \\
& \left.+e^{2}\left[\frac{1}{6}+\frac{5}{24} \sin ^{2} i-\frac{35 J_{4}}{18 J_{2}^{2}}\left(\frac{9}{7}-\frac{9}{4} \sin ^{2} i\right)\right]+\sqrt{1-e^{2}}\left(1-\frac{3}{2} \sin ^{2} i\right)\right\} \cos i
\end{aligned}
$$

Sun-synchronous orbits require the precession rate of ascending node to be equal to the Saturn rotational angular speed around the Sun. Hence, the average nodal precession rate is 


$$
\dot{\bar{\Omega}}=\dot{\Omega}_{1}+\dot{\Omega}_{2}=n_{s} .
$$

Here $n_{s}$ is the mean angular velocity around the Sun. Thus, we gained the equation from Equation (3):

$$
f(\cos i)=A \cos ^{3} i+B \cos i+C=0,
$$

where

$$
\begin{aligned}
& A=\frac{9 n J_{2}^{2} R_{e}^{4}}{4 p^{4}}\left[e^{2}\left(-\frac{5}{24}-\frac{35 J_{4}}{8 J_{2}^{2}}\right)+\frac{3}{2} \sqrt{1-e^{2}}+\left(\frac{5}{3}-\frac{35 J_{4}}{12 J_{2}^{2}}\right)\right], \\
& B=\frac{3 n J_{2} R_{e}^{2}}{2 p^{2}}\left\{1+\frac{3 J_{2} R_{e}^{2}}{2 p^{2}}\left[e^{2}\left(\frac{3}{8}+\frac{15 J_{4}}{8 J_{2}^{2}}\right)-\frac{1}{2} \sqrt{1-e^{2}}-\left(\frac{1}{6}-\frac{5 J_{4}}{4 J_{2}^{2}}\right)\right]\right\} \\
& C=n_{s} .
\end{aligned}
$$

For sun-synchronous orbits, we can derive the inequality systems (5) to calculate the inclination from theoretical analysis. The following inequalities are the requirements to find the roots of $\dot{\bar{\Omega}}=n_{s}$.

$$
\left\{\begin{aligned}
1-R_{e} / a & >e \\
\frac{C^{2}}{4 A^{2}}+\frac{B^{3}}{27 A^{3}} & \leq 0
\end{aligned}\right.
$$

The first inequality in (5) prevents the spacecraft from crashing into the outer atmosphere of Saturn. The second inequality is the discriminant of Equation (4). Then we obtain the plot of the discriminant, Figure 1.

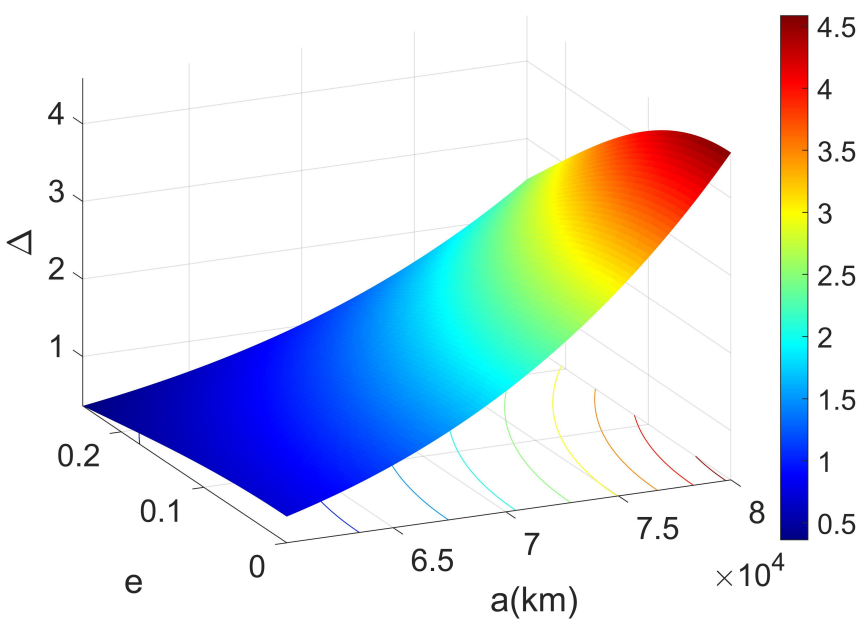

Figure 1. The discriminant of sun-synchronous orbits for different values of semimajor axis and eccentricity.

We learn from Figure 1 that the discriminant is almost always positive for any chosen $a$ and $e$. It means Equation (4) only has one real root, and there is only one sun-synchronous orbit for any chosen semimajor axis and eccentricity. Here, we choose the orbital elements $a=62,268 \mathrm{~km}$ and $e=0.01$, substitute them into Equation (4), and solve the equation. Then the inclination $i=90.0483 \mathrm{deg}$ can be obtained naturally.

Here we continue to analyze the perturbations of spacecraft in circular sun-synchronous orbits. The spacecraft located in circular sun-synchronous orbits around Saturn would be perturbed by the Sun. The derivative of inclination is presented by using the Lagrange equations as $[33,34]$ : 


$$
\begin{aligned}
\frac{d i}{d t}= & \frac{r \cos (\omega+f)}{n a^{2} \sqrt{1-e^{2}}} F_{n} \\
\approx & \frac{3 n_{s}^{2} \cos (\omega+f) \cos \xi}{n}\left(\cos \beta_{s} \sin i \sin \Omega-\sin \beta_{s} \cos i_{s} \sin i \cos \Omega+\sin \beta_{s} \sin i_{s} \cos i\right), \\
\cos \xi= & \cos \beta_{s}[\cos (\omega+f) \cos \Omega-\cos i \sin (\omega+f) \sin \Omega]+\sin \beta_{s} \cos i_{s}[\cos (\omega+f) \sin \Omega \\
& +\cos i \sin (\omega+f) \cos \Omega]+\sin \beta_{s} \sin i_{s} \sin i \sin (\omega+f),
\end{aligned}
$$

where $F_{n}$ is the normal perturbation force caused by the solar gravitation, $n_{s}$ is the angular speed of Saturn around the Sun, $\beta_{S}$ is the ecliptic longitude of the Sun, $i_{S}$ is the obliquity of the ecliptic of Saturn, and $\xi$ is the angle between the unit vector from center of mass of Saturn pointing the spacecraft and the unit vector from center of mass of Saturn pointing at the Sun. Then we average the secular derivative of inclination in a period of circular sun-synchronous orbits [35]. We could see the secular inclination perturbation as:

$$
\frac{\overline{d i}}{d t}=-\frac{3 n_{s}^{2}}{16 n} \sin i\left(1+\cos i_{s}\right)^{2} \sin \left(2 \beta_{s}-2 \Omega\right)
$$

It is important to emphasize that we only consider the secular effect of perturbations. We can substitute $\frac{d i}{d t}$ for $\frac{\overline{d i}}{d t}$ for convenience. $\left(2 \beta_{s}-2 \Omega\right)$ in Equation (6) is associated with the local time drift at the descending node.

The semimajor axis decay due to atmospheric drag leads to a shorter period and damage of the recursive feature. Here we simply assume the atmosphere of Saturn is stationary. According to the preliminary study of Saturn atmosphere model [8], we could estimate the acceleration of semi-major axis caused by atmospheric drag. We describe the variation of semimajor axis due to atmospheric drag of circular orbits by $[29,36]$

$$
\frac{d a}{d t}=-\frac{S C_{d} \rho n a^{2}}{m}
$$

where $C_{d}$ is the drag coefficient, $S$ is the projected area, $\rho$ is the neutral atmosphere density, and $m$ is the mass of spacecraft.

When we analyze the inclination perturbation, we can calculate the local time drift at the descending node instead. Meanwhile, it is a fact that the local time drift at the descending node is also the local time drift at ascending node. Thus, we could continue to analyze the evolution of the ascending node. According to Equation (3), the first-order approximation of $\overline{\bar{\Omega}}$ by using first-order Taylor expansion is

$$
\Delta \dot{\bar{\Omega}}=\frac{\partial \Delta \dot{\bar{\Omega}}}{\partial a} \Delta a+\frac{\partial \Delta \dot{\bar{\Omega}}}{\partial i} \Delta i
$$

where

$$
\begin{gathered}
\left\{\begin{array}{l}
\Delta a=\Delta a_{0}+\frac{d a}{d t}\left(t-t_{0}\right) \\
\Delta i=\Delta i_{0}+\frac{d i}{d t}\left(t-t_{0}\right)
\end{array}\right. \\
\frac{\partial \dot{\bar{\Omega}}}{\partial a}=\frac{21 n J_{2} R_{e}^{2} \cos i}{4 p^{3}}\left(1-e^{2}\right)+\frac{99 n J_{2}^{2} R_{e}^{4}}{8 p^{5}}\left(1-e^{2}\right)\left\{\left[\frac{3}{2}-\frac{5}{3} \sin ^{2} i-\frac{35 J_{4}}{18 J_{2}^{2}}\left(\frac{6}{7}-\frac{3}{2} \sin ^{2} i\right)\right]\right. \\
\left.+\left[\frac{1}{6}+\frac{5}{24} \sin ^{2} i-\frac{35 J_{4}}{18 J_{2}^{2}}\left(\frac{9}{7}-\frac{9}{4} \sin ^{2} i\right)\right] e^{2}+\left(1-\frac{3}{2} \sin ^{2} i\right) \sqrt{1-e^{2}}\right\} \cos i \\
\frac{\partial \dot{\bar{\Omega}}}{\partial i}=\frac{3 n J_{2} R_{e}^{2} \sin i}{2 p^{2}}-\frac{9 n J_{2}^{2} R_{e}^{4}}{4 p^{4}}\left\{\left[\left(-\frac{29}{6}+\frac{15 J_{4}}{2 J_{2}^{2}}\right) \sin i+\left(5-\frac{35 J_{4}}{4 J_{2}^{2}}\right) \sin ^{3} i\right]\right. \\
\left.+\left[\left(\frac{1}{4}+\frac{45 J_{4}}{4 J_{2}^{2}}\right) \sin i-\left(\frac{5}{8}+\frac{105 J_{4}}{8 J_{2}^{2}}\right) \sin ^{3} i\right] e^{2}+\left(-4 \sin i+\frac{9}{2} \sin ^{3} i\right) \sqrt{1-e^{2}}\right\}
\end{gathered}
$$


For Saturn, because the rotation period is $10.656 \mathrm{~h}, 1 \mathrm{deg}$ drift of the ascending node results in 1.776 min drift of spacecraft. Then according to Equation (8), the local time drift of spacecraft at ascending node is:

$$
\begin{aligned}
\Delta T= & \frac{1.776 \times 180}{\pi} \Delta \Omega(t) \\
= & \frac{1.776 \times 180}{\pi}\left[\Delta \Omega\left(t_{0}\right)+\frac{\partial \dot{\bar{\Omega}}}{\partial a} \Delta a\left(t_{0}\right)\left(t-t_{0}\right)+\frac{1}{2} \frac{\partial \dot{\bar{\Omega}}}{\partial a} \frac{d a}{d t}\left(t-t_{0}\right)^{2}\right. \\
& \left.+\frac{\partial \dot{\bar{\Omega}}}{\partial i} \Delta i\left(t_{0}\right)\left(t-t_{0}\right)+\frac{1}{2} \frac{\partial \overline{\bar{\Omega}}}{\partial i} \frac{d i}{d t}\left(t-t_{0}\right)^{2}\right] .
\end{aligned}
$$

The unit of spacecraft running time is second. The local time drift is counted in minutes.

When we only investigate the local time drift caused by solar gravitation, Equation (9) could be rewritten as

$$
\begin{aligned}
\Delta T= & \frac{1.776 \times 180}{\pi}\left[\Delta \Omega\left(t_{0}\right)+\frac{\partial \dot{\bar{\Omega}}}{\partial a} \Delta a\left(t_{0}\right)\left(t-t_{0}\right)\right. \\
& \left.+\frac{\partial \dot{\bar{\Omega}}}{\partial i} \Delta i\left(t_{0}\right)\left(t-t_{0}\right)+\frac{1}{2} \frac{\partial \overline{\bar{\Omega}}}{\partial i} \frac{d i}{d t}\left(t-t_{0}\right)^{2}\right]
\end{aligned}
$$

There are four initial variables, $\Delta \Omega\left(t_{0}\right), \Delta a\left(t_{0}\right), \Delta i\left(t_{0}\right)$, and $-\left(\beta_{s}-\Omega\right)$ in Equation (10). In this paper, we assume $\Delta \Omega\left(t_{0}\right)=0$ and only consider the effect of other three variables of a given sun-synchronous orbit around Saturn.

The initial orbital elements of the circular sun-synchronous orbits given in this paper are $a=62,268 \mathrm{~km}, e=0.01, i=90.0483 \mathrm{deg}, \Omega=-45 \mathrm{deg}, \omega=30 \mathrm{deg}$, and $M=0 \mathrm{deg}$. The ecliptic of longitude of the Sun is fixed at $\beta_{s}=90 \mathrm{deg}$.

When we choose small initial orbital deviation $\Delta a_{0}=1 \mathrm{~km}, \Delta i_{0}=0.001 \mathrm{deg}$, and arbitrary $\Omega \in[0,360] \mathrm{deg}$, we could know the local time drift at any descending node after 10 Earth years in Figure $2 a$. In Figure $2 a$, in the range of $-\left(\beta_{s}-\Omega\right) \in[0,90] \cup[180,270]$ deg, the local time drift $\Delta T$ is positive, which means the local time at descending node has been delayed. In the range $-\left(\beta_{s}-\Omega\right) \in[90,180] \cup[-90,0] \mathrm{deg}$, the local time drift $\Delta T$ is negative, which means the local time at the descending node has been brought forward. When the initial inclinaiton deviation is slightly greater, the local time in a larger range of $-\left(\beta_{s}-\Omega\right)$ in Figure $2 \mathrm{~b}$ is delayed. On the contrary, when we choose a negative initial inclination deviation, the absolute value is equal to the initial inclination deviation of Figure $2 b$, the local time of more area of Figure $2 c$ is brought forward. Furthermore, we could still observe from Figure $2 \mathrm{a}-\mathrm{c}$ that the distribution of the extremum of local time drift is fixed, regardless of whether and how the value and sign of $-\left(\beta_{s}-\Omega\right)$ change. To reduce the influence of different choices of initial condition, we choose the value of $-\left(\beta_{s}-\Omega\right)$ where the local time drift will reach the extremum after 10 Earth years. Therefore, in this paper, we fix the value $-\left(\beta_{s}-\Omega\right)=-135 \mathrm{deg}$, which means the local time at the descending node is 15:00.

When we fix the initial inclination deviation and choose different initial semimajor axis deviation, comparing Figure $2 \mathrm{~b}$ with Figure $2 \mathrm{~d}$, the extremum of local time drift of these two figures almost have the same distribution. This means the first-order approximation of local time drift is sensitive to the initial inclination deviation and insensitive to the initial semimajor axis deviation. Thus, we fix the semimajor axis deviation in this paper $\Delta a_{0}=1 \mathrm{~km}$.

When we consider the solar gravitation perturbation and the atmospheric drag at the same time, we should use Equation (9) to calculate the local time drift at the descending node. The fact we should notice is that the changing trend of $\frac{d a}{d t}$ and $\frac{d i}{d t}$ is quadratic function of time $t$ [29]. Therefore, we try to simulate the effect of atmospheric drag by using the inclinaiton drift caused by solar gravity. In this way, we only need to choose inclination 
prebiased methods to offset the local time drift caused by two different kinds of force. The explicit calculation of local time drift:

$$
\begin{aligned}
\Delta \tilde{T}= & \frac{1.776 \times 180}{\pi} \Delta \Omega(t) \\
= & \frac{1.776 \times 180}{\pi}\left\{\Delta \Omega\left(t_{0}\right)+\left(\frac{\partial \dot{\bar{\Omega}}}{\partial a} \frac{d a}{d t} / \frac{\partial \dot{\bar{\Omega}}}{\partial i} \frac{d i}{d t}+1\right)\right. \\
& {\left.\left[\frac{\partial \dot{\bar{\Omega}}}{\partial i} \Delta i\left(t_{0}\right)\left(t-t_{0}\right)+\frac{1}{2} \frac{\partial \overline{\bar{\Omega}}}{\partial i} \frac{d i}{d t}\left(t-t_{0}\right)^{2}\right]\right\} . }
\end{aligned}
$$

We find two figures to test the effect of simulating method used in Equation (11). In each figure, there are two curves representing the local time drift in 5 years in different calculation methods. The curves which have considered atmospheric drag use Equation (9). The curves simulating the atmospheric drag use Equation (11). Comparing Figure 3a with Figure 3b, we learn that the circular sun-synchronous orbits with the smaller initial inclination deviation has the better simulating effect of atmospheric drag.

Then we use two methods in this paper to offset the local time drift at the descending node.

The first control strategy is using initial inclination biased method. This control strategy suits the spacecraft whose entire lifetime is not too long. We take an initial inclination bias to make the spacecraft located in a quasi-sun-synchronous orbit. Then the inclination of the quasi-sun-synchronous orbit will oscillate around its normal inclination in its lifetime but still maintain the feature of sun-synchronous orbits.

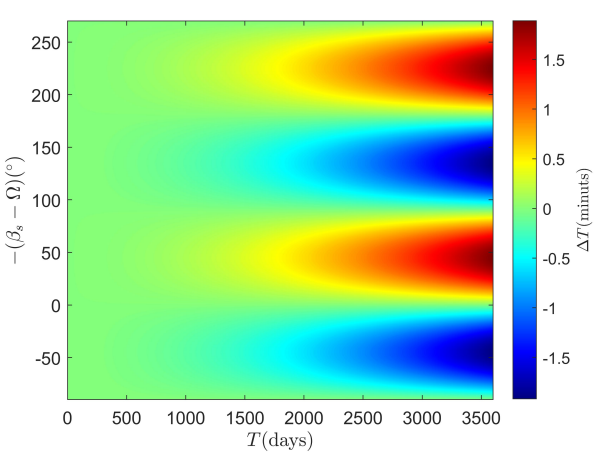

(a)

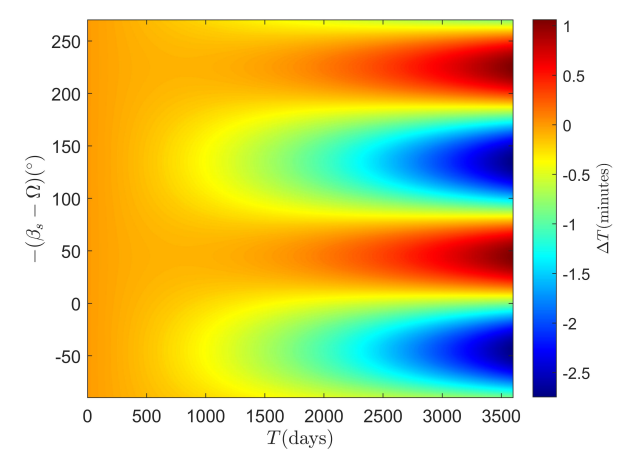

(c)

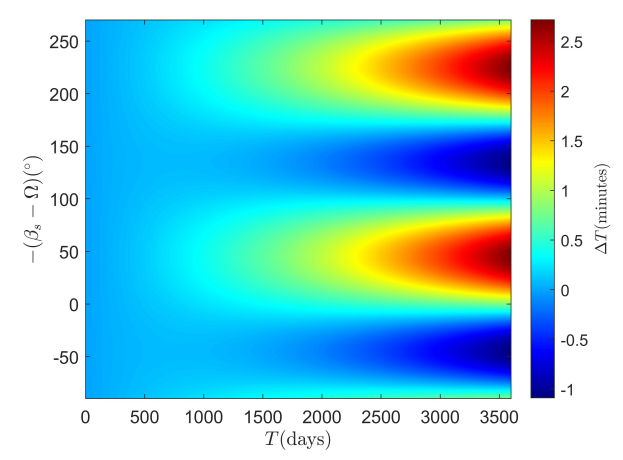

(b)

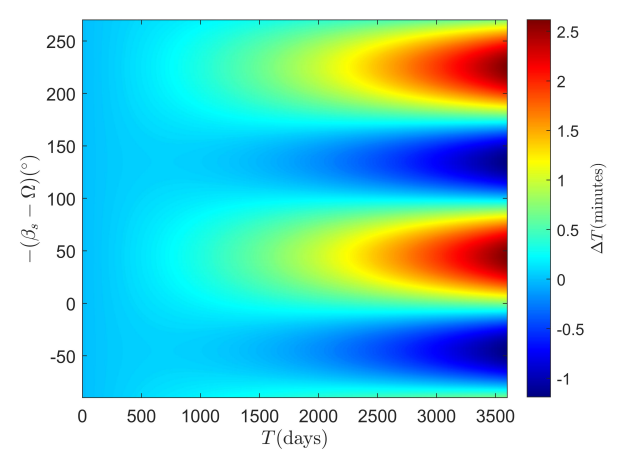

(d)

Figure 2. Four initial conditions of the local time drift at descending node in 10 Earth years with solar gravitational perturbation effect: (a) $\Delta a_{0}=1 \mathrm{~km}, \Delta i_{0}=0 \mathrm{deg}, \Omega \in[0,360] \mathrm{deg}$, $\beta_{s}=90 \mathrm{deg} ;$ (b) $\Delta a_{0}=1 \mathrm{~km}, \Delta i_{0}=3 \times 10^{-4} \mathrm{deg}, \Omega \in[0,360] \mathrm{deg}, \beta_{s}=90 \mathrm{deg}$; (c) $\Delta a_{0}=1 \mathrm{~km}$, $\Delta i_{0}=-3 \times 10^{-4} \mathrm{deg}, \Omega \in[0,360] \mathrm{deg}, \beta_{s}=90 \mathrm{deg} ;$ and (d) $\Delta a_{0}=10 \mathrm{~km}, \Delta i_{0}=3 \times 10^{-4} \mathrm{deg}$, $\Omega \in[0,360] \mathrm{deg}, \beta_{s}=90 \mathrm{deg}$. 
When $t_{e}=-\frac{\Delta i\left(t_{0}\right)}{\frac{d i}{d t}}$, the local time drift $\Delta \tilde{T}$ in Equation (11) sees its extremum

$$
\Delta \tilde{T}\left(t_{e}\right)=-\frac{3996}{25 \pi} \frac{\partial \dot{\bar{\Omega}}}{\partial i} \frac{\Delta i\left(t_{0}\right)^{2}}{\frac{d i}{d t}}
$$

If the end of lifetime of the spacecraft is $t_{f}$, then the limit of local time drift at descending node is $|\Delta \tilde{T}(t f)| \leq \Delta \tilde{T}\left(t_{e}\right)$. Let $\Delta \tilde{T}\left(t_{f}\right)=-\Delta \tilde{T}\left(t_{e}\right)$, then we find the initial inclination bias:

$$
\Delta i\left(t_{0}\right)=(1-\sqrt{2}) \frac{d i}{d t} t_{f}
$$

From Figure $3 c, d$, we learn that the prebiased initial strategy damps the local time drift at the descending node in the designed 5 Earth years' lifetime of spacecraft.

The second control strategy is designed for the spacecraft which need to execute a long-time mission. This method needs periodic inclination bias to damp the local time drift caused by solar gravitation. The extremum of local time drift Equation (11) in a period $t_{c}=-2 \frac{\Delta i\left(t_{c_{0}}\right)}{\frac{d i}{d t}}$ is equal to a fixed local time drift limitation $\Delta \tilde{T}$

$$
\left|\Delta \tilde{T}\left(\frac{t_{c}}{2}\right)\right|=|\Delta \tilde{T}|
$$

use the calculation equation in

$$
\frac{1.776 \times 180}{\pi}\left[\Delta \Omega\left(t_{0}\right)-\frac{1}{2}\left(\frac{\partial \dot{\bar{\Omega}}}{\partial a} \frac{d a}{d t} / \frac{\partial \dot{\bar{\Omega}}}{\partial i} \frac{d i}{d t}+1\right) \frac{\partial \dot{\bar{\Omega}}}{\partial i} \frac{\Delta i\left(t_{c_{0}}\right)^{2}}{\frac{d i}{d t}}\right]=|\Delta \tilde{T}|
$$

when $\Delta \Omega\left(t_{0}\right)=0$, then we find

$$
-\frac{3996}{25 \pi}\left(\frac{\partial \dot{\bar{\Omega}}}{\partial a} \frac{d a}{d t} / \frac{\partial \dot{\bar{\Omega}}}{\partial i} \frac{d i}{d t}+1\right) \frac{\partial \dot{\bar{\Omega}}}{\partial i} \frac{\Delta i\left(t_{c_{0}}\right)^{2}}{\frac{d i}{d t}}=|\Delta \tilde{T}|
$$

and the value of periodic inclination bias can be derived naturally:

$$
\left|\Delta i\left(t_{c_{0}}\right)\right|=\sqrt{\left|\frac{25 \pi}{3996 \frac{\partial \dot{\bar{\Omega}}}{\partial i}\left(\frac{\partial \dot{\Omega}}{\partial a} \frac{d a}{d t} / \frac{\partial \tilde{\bar{\Omega}}}{\partial i} \frac{d i}{d t}+1\right)}\right|}
$$

where the sign of $\Delta i\left(t_{c_{0}}\right)$ is opposite to the sign of $\frac{d i}{d t}$.

From Figure $3 c, d$, compared with Figure $4 a, b$, the periodic inclination biased method has the better effect of local time drift limitation. Meanwhile, comparing Figure $4 \mathrm{a}$ with Figure $4 b$, it is obviously also sensitive to the initial inclination deviation. Therefore, regardless of the methods, it is essential to limit the initial inclination deviation $\Delta i_{0}$. 


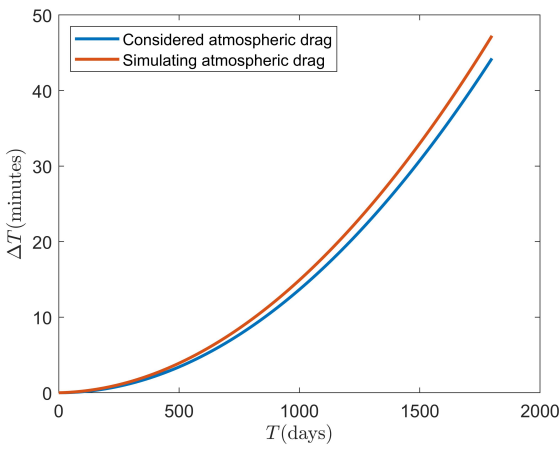

(a)

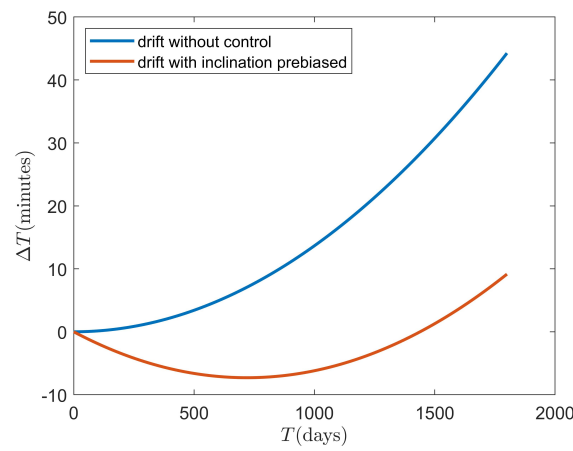

(c)

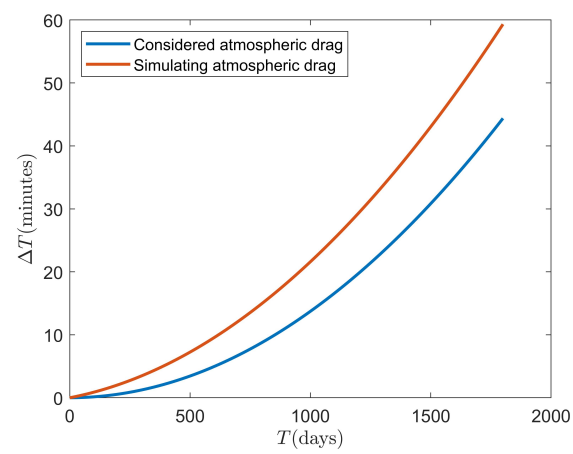

(b)

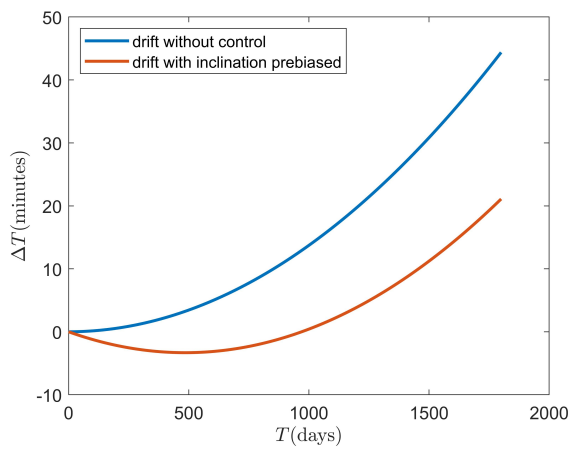

(d)

Figure 3. A comparison between $\Delta T$ considered atmospheric drag and $\Delta \tilde{T}$ simulating the atmospheric drag in 5 Earth years (a) $\Delta a_{0}=1 \mathrm{~km}, \Delta i_{0}=1 \times 10^{-5} \mathrm{deg},-\left(\beta_{s}-\Omega\right)=-135 \mathrm{deg}$; (b) $\Delta a_{0}=1 \mathrm{~km}$, $\Delta i_{0}=1 \times 10^{-4} \mathrm{deg},-\left(\beta_{s}-\Omega\right)=-135 \mathrm{deg}$. A comparison between $\Delta T$ considered atmospheric drag and $\Delta \tilde{T}$ simulating the atmospheric drag with a prebiased initial inclination in 5 Earth years (c) $\Delta a_{0}=1 \mathrm{~km}, \Delta i_{0}=1 \times 10^{-5} \mathrm{deg},-\left(\beta_{s}-\Omega\right)=-135 \mathrm{deg} ;(\mathbf{d}) \Delta a_{0}=1 \mathrm{~km}, \Delta i_{0}=1 \times 10^{-4} \mathrm{deg}$, $-\left(\beta_{s}-\Omega\right)=-135 \mathrm{deg}$.

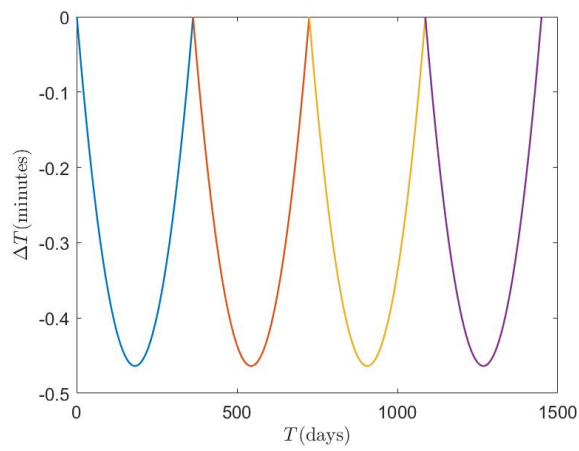

(a)

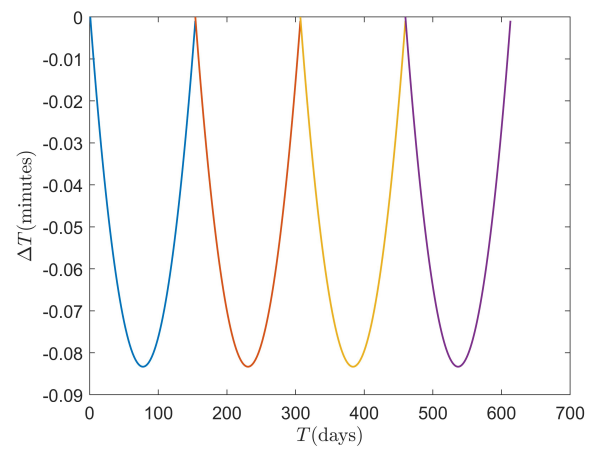

(b)

Figure 4. The local time drift $\Delta \tilde{T}$ after four inclination biased periods when the limited extremum of local time drift $\Delta T_{L}=0.1 \mathrm{~min}$ (a) $\Delta a_{0}=1 \mathrm{~km}, \Delta i_{0}=1 \times 10^{-5} \mathrm{deg},-\left(\beta_{s}-\Omega\right)=-135 \mathrm{deg}$, control period $T_{P}=363$ days; (b) $\Delta a_{0}=1 \mathrm{~km}, \Delta i_{0}=5 \times 10^{-5} \mathrm{deg},-\left(\beta_{S}-\Omega\right)=-135 \mathrm{deg}$, control period $T_{P}=154$ days. 


\section{Repeating Ground Track Orbits and Orbital Maintenance}

The repeating ground track is significant for remote sensing satellites. The condition for spacecraft achieving repeating ground track is $R \Delta \lambda=2 \pi N$, where $R$ and $N$ are relatively positive prime numbers. The interval of the adjacent ground track on the equator is $[28,29]$

$$
\Delta \lambda=T_{N}\left(\omega_{S}-\dot{\bar{\Omega}}\right),
$$

which $\omega_{S}$ is the angular rotational velocity of Saturn.

The nodal period of the motion of spacecraft is

$$
T_{N}=\frac{2 \pi}{\dot{\bar{M}}+\dot{\bar{\omega}}} .
$$

According to the mean element theory [16], the average perturbing rates of $M$ and $\omega$ is [37]

$$
\begin{aligned}
\dot{\bar{M}} & =n+\dot{M}_{1}+\dot{M}_{2}, \\
\dot{\bar{\omega}} & =\dot{\omega}_{1}+\dot{\omega}_{2},
\end{aligned}
$$

where

$$
\begin{gathered}
\dot{M}_{1}=\frac{3 n J_{2} R_{e}^{2}}{2 p^{2}}\left(1-\frac{3}{2} \sin ^{2} i\right) \sqrt{1-e^{2}}, \\
\dot{\omega}_{1}=-\frac{3 n J_{2} R_{e}^{2}}{2 p^{2}}\left(\frac{5}{2} \sin ^{2} i-2\right), \\
\dot{M}_{2}=\frac{9 n J_{2}^{2} R_{e}^{4}}{8 p^{4}}\left(1-e^{2}\right)\left(1-\frac{3}{2} \sin ^{2} i\right)^{2} \\
+\frac{9 n J_{2}^{2} R_{e}^{4}}{4 p^{4}}\left\{\sqrt{1-e^{2}}\left(\frac{5}{2}-\frac{19}{3} \sin ^{2} i+\frac{233}{48} \sin ^{4} i\right)\right. \\
+\frac{e^{4}}{\sqrt{1-e^{2}}}\left(\frac{35}{12}-\frac{35}{4} \sin ^{2} i+\frac{315}{32} \sin ^{4} i\right) \\
\left.+\sqrt{1-e^{2}} e^{2}\left[\left(\frac{10}{3}-\frac{5 J_{4}}{4 J_{2}^{2}}\right)+\left(-\frac{26}{3}+\frac{25 J_{4}}{4 J_{2}^{2}}\right) \sin ^{2} i+\left(\frac{103}{12}-\frac{175 J_{4}}{32 J_{2}^{2}}\right) \sin ^{4} i\right]\right\}, \\
\dot{\omega}_{2}=\frac{9 n J_{2}^{2} R_{e}^{4}}{4 p^{4}}\left\{\left(4-\frac{10 J_{4}}{3 J_{2}^{2}}\right)+\left(-\frac{103}{12}+\frac{155 J_{4}}{12 J_{2}^{2}}\right) \sin ^{2} i+\left(\frac{215}{48}-\frac{245 J_{4}}{24 J_{2}^{2}}\right) \sin ^{4} i\right. \\
+\sqrt{1-e^{2}}\left(2-\frac{11}{2} \sin ^{2} i+\frac{15}{4} \sin ^{4} i\right)+e^{2}\left[\left(\frac{7}{12}-\frac{15 J_{4}}{4 J_{2}^{2}}\right)+\left(-\frac{3}{8}+\frac{105 J_{4}}{8 J_{2}^{2}}\right) \sin ^{2} i\right. \\
\left.\left.+\left(-\frac{15}{32}-\frac{315 J_{4}}{32 J_{2}^{2}}\right) \sin ^{4} i\right]\right\} .
\end{gathered}
$$

In this paper, we use the ground track repetition parameter $Q=R / N$ to describe different repeating ground track orbits. Here, we should pay attention to the meaningful range of repetition parameter $Q$ [29].

To find a suitable repetition parameter, we should firstly find the lower bound of $Q$ by using the discriminant of $f(\sin i)$ :

$$
f(\sin i)=A_{1} \sin ^{4} i+B_{1} \sin ^{2} i+C_{1}=0,
$$

where

$$
\begin{aligned}
A_{1}= & \frac{9 n J_{2}^{2} R_{e}^{4}}{4 p^{4}}\left[\left(\frac{215}{48}-\frac{15}{32} e^{2}+\frac{15}{4} \sqrt{1-e^{2}}\right)-\frac{35 J_{4}}{18 J_{2}^{2}}\left(\frac{21}{4}+\frac{81}{16} e^{2}\right)\right]+\frac{9 n J_{2}^{2} R_{e}^{4}}{4 p^{4}} \sqrt{1-e^{2}}\left(\frac{9}{8} \sqrt{1-e^{2}}\right. \\
& \left.+\frac{233}{48}+\frac{103}{12} e^{2}+\frac{315}{32} \frac{e^{4}}{1-e^{2}}-\frac{105 J_{4}}{32 J_{2}^{2}} e^{2}\right),
\end{aligned}
$$




$$
\begin{aligned}
B_{1}= & \frac{3 n J_{2} R_{e}^{2}}{4 p^{2}}\left\{-5+\frac{3 J_{2} R_{e}^{2}}{p^{2}}\left[-\frac{103}{12}-\frac{3}{8} e^{2}-\frac{11}{2} \sqrt{1-e^{2}}+\frac{35 J_{4}}{6 J_{2}^{2}}\left(\frac{31}{14}+\frac{9}{4} e^{2}\right)\right]\right\}-\frac{9 n J_{2} R_{e}^{2}}{4 p^{2}} \sqrt{1-e^{2}} \\
+ & \frac{9 n J_{2}^{2} R_{e}^{4}}{4 p^{4}} \sqrt{1-e^{2}}\left(-\frac{3}{2} \sqrt{1-e^{2}}-\frac{19}{3}-\frac{26}{3} e^{2}-\frac{35}{4} \frac{e^{4}}{1-e^{2}}+\frac{25 J_{4}}{4 J_{2}^{2}} e^{2}\right), \\
C_{1} & =\frac{3 n J_{2} R_{e}^{2}}{4 p^{2}}\left\{4+\frac{3 J_{2} R_{e}^{2}}{p^{2}}\left[\left(4+\frac{7}{12} e^{2}+2 \sqrt{1-e^{2}}\right)-\frac{5 J_{4}}{6 J_{2}^{2}}\left(4+\frac{9}{2} e^{2}\right)\right]\right\}+\frac{3 n J_{2} R_{e}^{2}}{2 p^{2}} \sqrt{1-e^{2}} \\
& +\frac{9 n J_{2} R_{e}^{4}}{4 p^{4}} \sqrt{1-e^{2}}\left(\frac{1}{2} \sqrt{1-e^{2}}+\frac{5}{2}+\frac{10}{3} e^{2}+\frac{35}{12} \frac{e^{4}}{1-e^{2}}-\frac{5 J_{4}}{4 J_{2}^{2}} e^{2}\right)+n+Q\left(n_{s}-\omega_{s}\right) .
\end{aligned}
$$

To promise $f(\sin i)$ has four real roots, the discriminant should be non-negative, i.e.,

$$
B_{1}^{2}-4 A_{1}\left[\tilde{C_{1}}-Q\left(\omega_{s}-n_{s}\right)\right] \geq 0 .
$$

Solving the inequality, we find the lower bound of $Q$ :

$$
Q \geq \frac{\tilde{C_{1}}-\frac{B_{1}^{2}}{4 A_{1}}}{\omega_{s}-n_{s}}
$$

where $\tilde{C_{1}}=C_{1}-Q\left(n_{s}-\omega_{s}\right)$.

To estimate the maximum of repetition parameter, we use the rotational period of Saturn $T_{S}$ and the period of spacecraft in a low orbit $T_{l}$. Then the upper bound of $Q$ can be presented as:

$$
Q \leq \frac{T_{s}}{T_{l}}
$$

Using the data from the planet model of Saturn, we find the meaningful range of repetition parameter for engineering application: $2.4701 \leq Q \leq 2.5191$.

For different chosen values of repetition parameter $Q$, we see a function $f(\sin i)$ that describes the relation between the inclination $i$ and the semimajor axis $a$ of repeating ground track orbits. The relation between $i$ and $a$ are shown in Figure 5 for three given meaningful values of $Q$. Using Equation (21), when initial semimajor axis and eccentricity are given, the corresponding inclination is the root of $f(\sin i)$. In Figure 6, we present the repeating ground track orbit around Saturn when initial orbital elements and repetition parameter $Q$ are given.

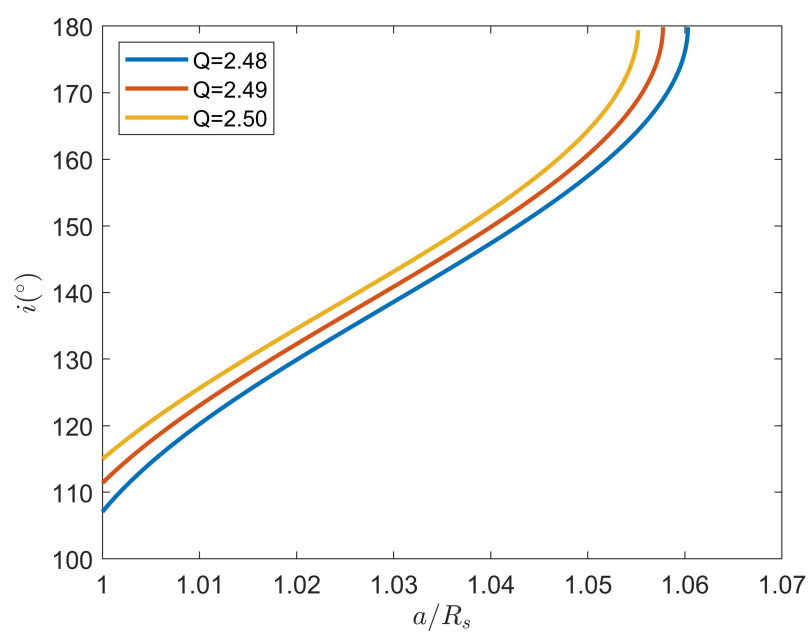

Figure 5. The relation between $a$ and $i$ of repeating ground track orbits for repetition parameter $Q=2.48,2.49$, and 2.50 . 


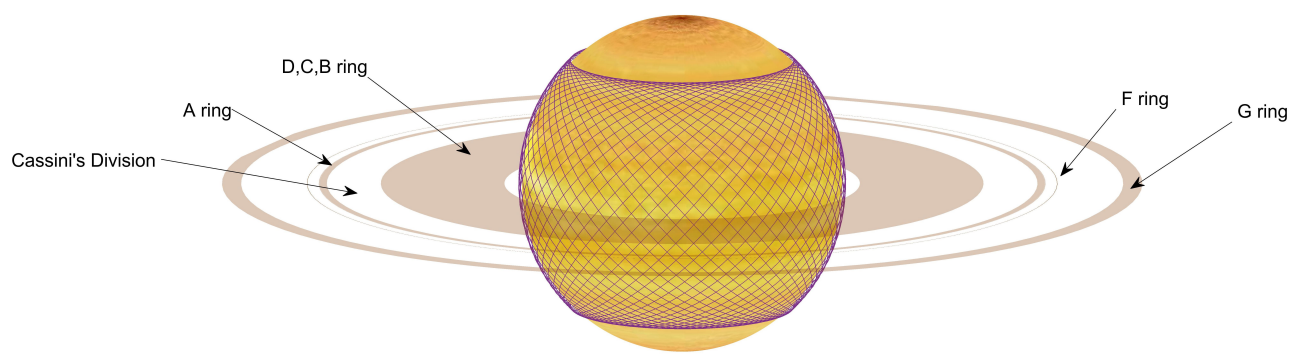

Figure 6. The repeating ground track orbit around Saturn for $a=61539 \mathrm{~km}, i=133.2052 \mathrm{deg}$, $e=0.001, \omega=45 \mathrm{deg}, \Omega=30 \mathrm{deg}$, and repetition parameter $Q=2.49$.

An inevitable perturbation which can cause the semimajor decay in repeating ground track orbits is atmospheric drag. The semimajor axis decay due to atmospheric drag leads to a shorter period and damage of the recursive feature.

The difference between actual angular velocity and nominal angular velocity can be presented as [29]:

$$
\Delta n=-\frac{3 \bar{n}}{2 \bar{a}}\left(a_{0}+\frac{d a}{d t} t-\bar{a}\right)
$$

where $a_{0}$ is the initial semimajor axis, $\bar{a}$ is the nominal semimajor axis, and $\bar{n}$ is nominal angular speed.

The increase in angular speed results in the longitude drift of ground track. Obviously, we can learn from Equation (7) that $\dot{a}<0$. Therefore, if $a_{0} \leq \bar{a}$, the longitude will drift eastward monotonously. To maintain the feature of repeating ground track orbits, we should take a maneuver to make initial semimajor axis $a_{0}>\bar{a}$. Then, in the first coming time interval $t_{s}=-\frac{\Delta a}{\dot{a}}$, the longitude will drift westward. When $\frac{d \Delta \lambda}{d t}=-\frac{3 \pi}{\bar{a}}(\Delta a+\dot{a} t)=0$, the longitude of the ground track would reach the western boundary. In the second time interval $t_{s}$, the longitude will drift eastward and finally return back to the initial longitude. Then we can find the longitude drift compared to the nominal repeating ground track orbits:

$$
\begin{aligned}
\Delta \lambda & =-\frac{\omega_{s}}{\bar{n}} \int_{0}^{t} \frac{3 \bar{n}}{2 \bar{a}}\left(a_{0}+\frac{d a}{d t} t-\bar{a}\right) d t \\
& =-\frac{3 \omega_{s}}{2 \bar{a}}\left[\left(a_{0}-\bar{a}\right) t+\frac{1}{2} \dot{a} t^{2}\right],
\end{aligned}
$$

where $\omega_{s}$ is the angular rotation speed of Saturnian.

In the whole control period, when we fix the limitation of longitude drift $\Delta \lambda_{L}$, then we can find the control period $\Delta T_{C}$ and compensation $\Delta a$ of semimajor axis [29]:

$$
\begin{aligned}
& \Delta \lambda_{L}=-\frac{3 \pi(\Delta a)^{2}}{2 \bar{a} \dot{a}}, \\
& \Delta a=\sqrt{-\frac{2 \bar{a} \dot{a} \Delta \lambda}{3 \pi}}, \\
& \Delta T_{C}=-\frac{2 \Delta a}{\dot{a}} .
\end{aligned}
$$

Thus, in each period, when the longitude of ground track drift to the eastern boundary, we have to take a maneuver $\Delta a$ to compensate the decay of semimajor axis. Here we assumed projected area of spacecraft $S=20 \mathrm{~m}^{2}, C_{d}=2.1, \Delta \lambda_{L}=10 \mathrm{~km}$, and mass $m=3000 \mathrm{~kg}$. The density of simplified atmospheric model, in the range of semi-major axis $a \in[62,268,62,468] \mathrm{km}$, is $\rho \in\left[3.7 \times 10^{-12}, 4.7 \times 10^{-12}\right] \mathrm{kg} / \mathrm{m}^{3}$ [8]. Then we find the Figures 7 and 8 which illustrate the the control compensation of semimajor axis and control period of repeating ground track orbits. From Figure 7, we know the semimajor compensation $\Delta a$ for $a \in[62,268,62,468] \mathrm{km}$ varies from $5200 \mathrm{~m}$ to $5800 \mathrm{~m}$. The corresponding compensation period $\Delta T_{C}$ in Figure 8 varies from $18 \mathrm{~h}$ to $16 \mathrm{~h}$. Compared to Jupiter [29], Saturnian atmospheric drag is about 2 orders of magnitude greater than Jovian atmospheric 
drag when the spacecraft is at the same altitude. As a result, it is essential to execute the semimajor axis compensation for repeating ground track orbits around Saturn.

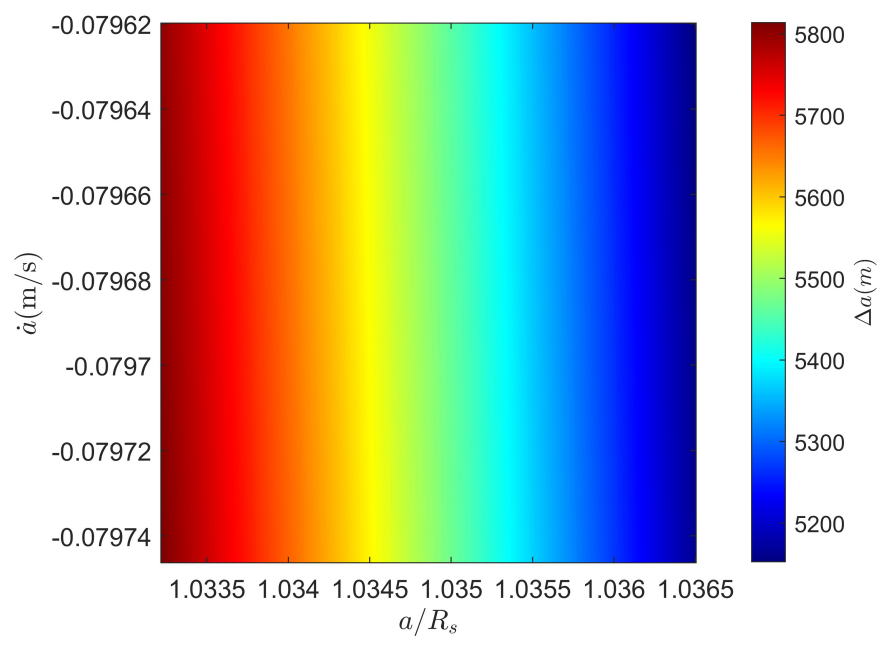

Figure 7. The semimajor aixs compensation $\Delta a(\mathrm{~m})$ for repeating ground track orbits where $a \in[62,268,62,468] \mathrm{km}$.

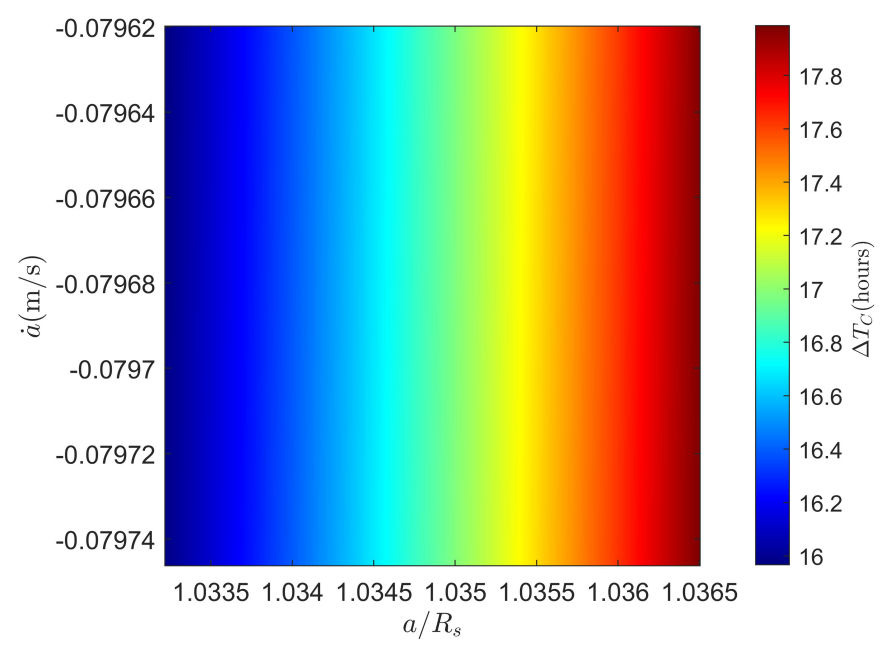

Figure 8. The semimajor aixs compensation period $\Delta T_{C}$ (hours) for repeating ground track orbits where $a \in[62,268,62,468] \mathrm{km}$.

\section{Frozen Orbits}

Before analyzing frozen orbits, we first investigate the orbits at the critical inclination. The traditional design of the orbits at critical inclination around Earth just considers the $J_{2}$ term by which the first order secular variation of eccentricity and argument of perigee are $[15,16]$ :

$$
\begin{aligned}
\dot{e}_{1} & =0, \\
\dot{\omega}_{1} & =-\frac{3 n J_{2} R_{2}^{2}}{2 a^{2}\left(1-e^{2}\right)^{2}}\left(\frac{5}{2} \sin ^{2} i-2\right) .
\end{aligned}
$$

Different from the methods which only considered $J_{2}$, we still need to calculate the perturbation caused by $J_{2}$ and $J_{4}$ terms. Though the magnitude of $J_{2}$ term exceeds $J_{3}$ term 1000 times, $J_{4}$ term still plays an important role in aspheric perturbation. Thus, the conditions for the orbits at the critical inclination should be combined $\omega_{1}$ with $\omega_{2}$ :

$$
\begin{aligned}
& \dot{e}_{1}=\dot{e}_{2}=0, \\
& \dot{\bar{\omega}}=\dot{\omega}_{1}+\dot{\omega}_{2}=0 .
\end{aligned}
$$


However, the critical inclination cannot satisfy most missions for remote sensing satellites. For the purpose of missions, we must consider the frozen orbits around Saturn. Frozen orbits are the orbits which own small constant eccentricity and constant argument of perigee while arbitrary inclination and orbiting altitude can be chosen. The frozen orbits we designed which have small eccentricity must consider first order long-period terms [23]. The main parts of long-period terms are [16]

$$
\begin{aligned}
\dot{e}_{1}^{l} & =-\frac{J_{3} R_{e}}{2 J_{2} p} \sin i\left(1-e^{2}\right) \sin \omega, \\
\dot{\omega}_{1}^{l} & =-\frac{J_{3} R_{e}}{2 e J_{2} p}\left(1-\frac{e^{2} \cos ^{2} i}{\sin i}\right) \cos \omega .
\end{aligned}
$$

Then the complete conditions for frozen orbits are combined by Equations (24) and (25) [23].

$$
\left\{\begin{array}{l}
\dot{\bar{e}}=\dot{e}_{1}+\dot{e}_{2}+\dot{e}_{1}^{l}=0, \\
\dot{\bar{\omega}}=\dot{\omega}_{1}+\dot{\omega}_{2}+\dot{\omega}_{1}^{l}=0 .
\end{array}\right.
$$

Using $\omega=90^{\circ}$ or $\omega=270^{\circ}$ to solve Equation (26), respectively, we learn that only when $\omega=270^{\circ}$ can the eccentricity $e$ be positive. In Figure 9, the values of eccentricity are presented for different combinations of semimajor axis and inclianition.

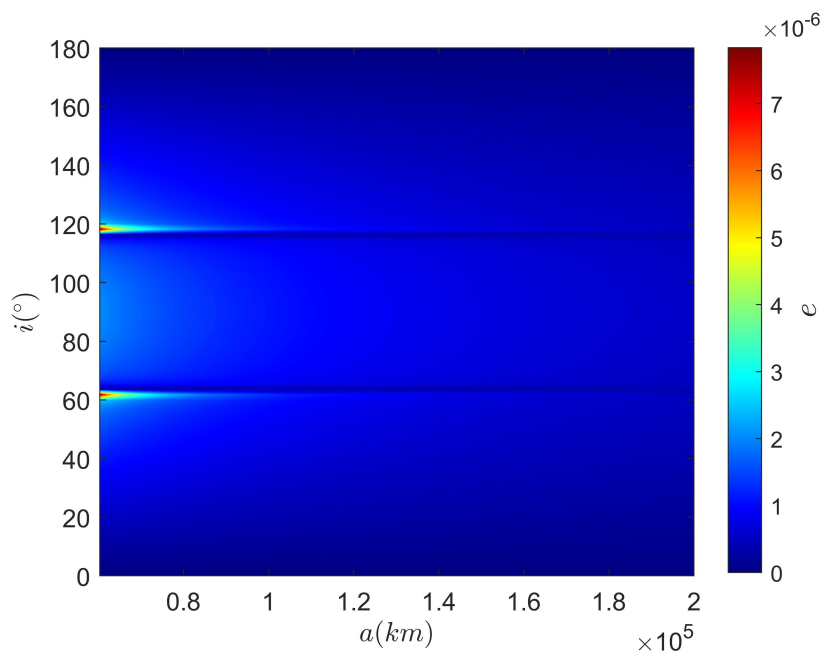

Figure 9. The eccentricity $e$ for different value of semi major axis $a$ and inclination $i$ when $\omega=270 \mathrm{deg}$.

\section{Stationary Orbits and Orbital Maintenance}

Stationary orbits are more complex than those we have considered before. Therefore, we investigate stationary orbits in a special condition. When we investigate the stationary orbits around terrestrial planets, we must take the $J_{22}$ term into our gravitational field model and analyze the longitude drift caused by aspheric perturbation. Here we only analyze aspheric perturbation caused by the $J_{2}, J_{4}$ terms, as Saturn is exactly a gaseous planet which means the $J_{22}$ term could be neglected.

The spherical coordinates $O-r, \lambda, \varphi$, where $O$ is the centroid of Saturn, $r$ is the distant from the spacecraft instant position to $O, \lambda$ and $\varphi$ represent the longitude and latitude of the spacecraft separately. The equations of motion of stationary orbits in the spherical coordinates can be written as [28] 


$$
\left\{\begin{aligned}
\ddot{r}-r \cos ^{2} \varphi \dot{\lambda}^{2}-r \dot{\varphi}^{2}= & -\frac{\mu}{r^{2}}-\frac{3 \mu J_{2} R_{e}^{2}}{2 r^{4}}\left(3 \sin ^{2} \varphi-1\right) \\
& +\frac{5 \mu J_{4} R_{e}^{4}}{8 r^{6}}\left(35 \sin ^{4} \varphi-30 \sin ^{2} \varphi+3\right) \\
\frac{d}{d t}\left(r^{2} \dot{\lambda} \cos ^{2} \varphi\right)= & 0 \\
r \ddot{\varphi}+2 \dot{r} \dot{\varphi}+\frac{1}{2} r^{2} \sin (2 \varphi) \dot{\lambda}^{2}= & -\left[\frac{3 \mu J_{2} R^{2}}{2 r^{3}}+\frac{\mu J_{4} R^{4}}{8 r^{5}}\left(70 \sin ^{2} \varphi-30\right)\right] \sin 2 \varphi .
\end{aligned}\right.
$$

Spacecraft staying in stationary orbits around Saturn must satisfy these conditions [28]:

$$
\left\{\begin{array}{l}
\dot{r}=\ddot{r}=0, \\
\dot{\lambda}=n, \ddot{\lambda}=0, \\
\dot{\varphi}=\ddot{\varphi}=0 .
\end{array}\right.
$$

Then we gain the equation of stationary orbits at $\varphi=0$ as

$$
\frac{\mu}{r^{3}}+\frac{3 \mu J_{2} R^{2}}{2 r^{5}}-\frac{15 \mu J_{4} R^{4}}{8 r^{7}}=n^{2} .
$$

Solving Equation (28), we find the radius of stationary orbits around Saturn is $r_{s}=112,506.0294 \mathrm{~km}$.

After learning the structure of Saturn's rings [12], we know that spacecraft in the stationary orbit around Saturn may have a collision with the B-ring.

Using nonsingular elements $\left\{a, e_{x}=e \cos (\Omega+\omega), e_{y}=e \sin (\Omega+\omega), i_{x}=\sin i \sin \Omega\right.$, $\left.i_{y}=\sin i \cos \Omega, \lambda=\Omega+\omega+M\right\}$, we first calculate the secular solar gravitation perturbation of $i_{x}, i_{y}$ and $e_{x}, e_{y}$. When $\varphi=0$, the normal perturbation force caused by the solar gravitation can be written as [33]:

$$
F_{n}=3 r n_{s}^{2}\left(\sin \alpha \sin \beta_{s} \cos i_{s}+\cos \alpha \cos \beta_{s}\right) \sin \beta_{s} \sin i_{s},
$$

where $\alpha$ is the longitude of spacecraft in the stationary orbit, $n_{S}$ is the angular speed of Saturn around the Sun, $\beta_{S}$ is the ecliptic longitude of the Sun, $i_{S}$ is the obliquity of the ecliptic of Saturn. The derivative of $i_{x}, i_{y}$ perturbed by normal solar gravitation in a Saturnian rotation period are [33]

$$
\begin{aligned}
\frac{d i_{x}}{d t}= & \frac{3 n_{s}^{2}}{n}\left[\frac{1}{4} \sin ^{2} \beta_{s} \sin 2 i_{s}+\left(\frac{1}{8} \sin 2 \beta_{s} \cos i_{s}\right) i_{x},\right. \\
& \left.+\left(-\frac{1}{8} \cos ^{2} \beta_{s}-\frac{3}{8} \sin ^{2} \beta_{s} \cos i_{s}+\frac{1}{2} \sin \beta_{s} \sin i_{s}\right) i_{y}\right], \\
\frac{d i_{y}}{d t}= & \frac{3 n_{s}^{2}}{n}\left[\frac{1}{4} \sin 2 \beta_{s} \sin i_{s}-\left(\frac{1}{8} \sin 2 \beta_{s} \cos i_{s}\right) i_{x}\right. \\
& \left.+\left(\frac{1}{8} \cos ^{2} \beta_{s}+\frac{3}{8} \sin ^{2} \beta_{s} \cos i_{s}-\frac{1}{2} \sin \beta_{s} \sin i_{s}\right) i_{y}\right] .
\end{aligned}
$$

Here, we apply the Equations (30) and (31) to analyze the perturbation of the stationary orbit caused by solar gravitation, then we find the average rate of inclination vector perturbed by solar gravity in a sidereal orbit period: 


$$
\left\{\begin{aligned}
\frac{\Delta i_{x}}{\Delta t} & =\frac{1}{2 \pi} \int_{0}^{2 \pi} \frac{d i_{x}}{d t} d \beta_{s} \\
& =\frac{3 n_{s}^{2}}{n}\left[\left(-\frac{1}{16}-\frac{3}{16} \cos ^{2} i_{s}+\frac{1}{4} \sin ^{2} i_{s}\right) i_{y}+\frac{1}{4} \sin i_{s} \cos i_{s}\right], \\
\frac{\Delta i_{y}}{\Delta t} & =\frac{1}{2 \pi} \int_{0}^{2 \pi} \frac{d i_{y}}{d t} d \beta_{s} \\
& =\frac{3 n_{s}^{2}}{n}\left(\frac{1}{16}+\frac{3}{16} \cos ^{2} i_{s}-\frac{1}{4} \sin ^{2} i_{s}\right) i_{x} .
\end{aligned}\right.
$$

After solving this first order linear ordinary differential equation system Equation (32), we find the $i_{x}(t), i_{y}(t)$ for any time $t$ with initial condition $i_{x}\left(t_{0}\right), i_{y}\left(t_{0}\right)$.

$$
\begin{aligned}
i_{x}(t)= & i_{x}\left(t_{0}\right) \cos \left[\frac{3 n_{s}^{2}}{32 n}\left(7 \cos \left(2 i_{s}\right)+1\right) t\right] \\
& -\left(i_{y}\left(t_{0}\right)-\frac{4 \sin \left(2 i_{s}\right)}{7 \cos \left(2 i_{s}\right)+1}\right) \sin \left[\frac{3 n_{s}^{2}}{32 n}\left(7 \cos \left(2 i_{s}\right)+1\right) t\right] \\
= & \left(0.621896-i_{y}\left(t_{0}\right)\right) \sin \left(1.35686 \times 10^{-13} t\right)+i_{x}\left(t_{0}\right) \cos \left(1.35686 \times 10^{-13} t\right), \\
i_{y}(t)= & i_{x}\left(t_{0}\right) \sin \left[\frac{3 n_{s}^{2}}{32 n}\left(7 \cos \left(2 i_{s}\right)+1\right) t\right] \\
& +\left(i_{y}\left(t_{0}\right)-\frac{4 \sin \left(2 i_{s}\right)}{7 \cos \left(2 i_{s}\right)+1}\right) \cos \left[\frac{3 n_{s}^{2}}{32 n}\left(7 \cos \left(2 i_{s}\right)+1\right) t\right]+\frac{4 \sin \left(2 i_{s}\right)}{7 \cos \left(2 i_{s}\right)+1} \\
= & 0.621896+i_{x}\left(t_{0}\right) \sin \left(1.35686 \times 10^{-13} t\right) \\
& +\left(i_{y}\left(t_{0}\right)-0.621896\right) \cos \left(1.35686 \times 10^{-13} t\right) .
\end{aligned}
$$

Before we analyze the perturbation of inclination vector, we firstly set the maximum of inclination perturbation $\left|\Delta i_{\max }\right|=0.2865^{\circ}$. Then we could draw the Figure 10a,b of $i_{x}, i_{y}$ separately for each initial condition value $i_{x_{0}}=i_{x}\left(t_{0}\right), i_{y_{0}}=i_{y}\left(t_{0}\right)$ in the limited circle. Once we see Figure 10a,b, what we know immediately is that the inclination perturbation is so small that the magnitude is smaller than $1 \times 10^{-3}$ in 5 Earth years. Furthermore, comparing Figure 10a with Figure 10b, we learn evidently that the magnitude of $\Delta i_{y}$ is two order magnitude smaller than $\Delta i_{x}$ in a given time of 5 Earth years. This means when we plot the perturbation of inclination vector of stationary orbit around Saturn over a long time, we could hardly see a sight difference in $i_{y}$.

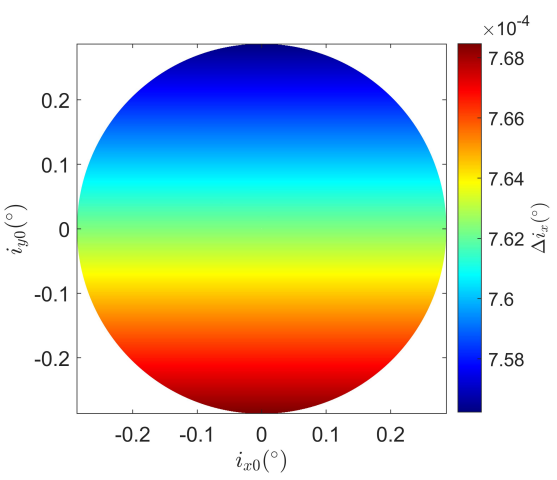

(a)

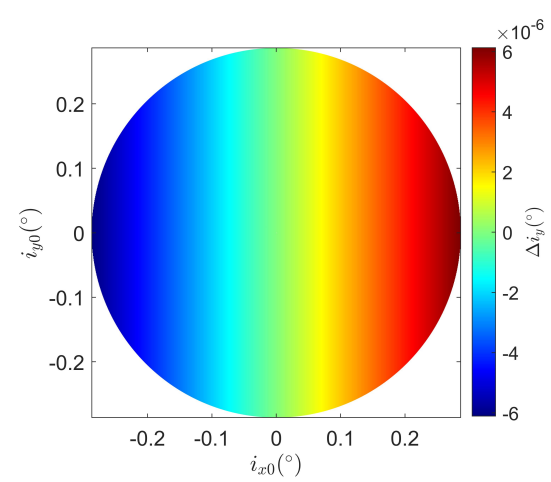

(b)

Figure 10. (a) $\Delta i_{x}$ (b) $\Delta i_{y}$ after 5 Earth years with initial inclination vector point $\left(i_{x_{0}}, i_{y_{0}}\right)$ in the inclination limited circle whose radius $r=0.2865 \mathrm{deg}$ perturbed by solar gravity.

Though the inclination perturbation caused by solar gravity could hardly influence the spacecraft in stationary orbit around Saturn, we still analyze the control strategy of $i_{x}, i_{y}$ in a preliminary way. When we know how the solar gravity perturbation acts, we could calculate $\Delta i$ for any initial $\left(i_{x_{0}}, i_{y_{0}}\right)$ to stay in the limited circle. The area of the cool 
tone circle overlap limited circle in Figure 11 indicates the initial point $\left(i_{x_{0}}, i_{y_{0}}\right)$ in this area are still remained in the limited circle after 100 Earth days. By observing the perturbation feature of stationary orbit around Saturn, we know that minimum maneuver $\overrightarrow{\Delta i}$ to keep $i_{x}, i_{y}$ staying in the limited circle is from the boundary of limited circle pointing towards the center of the cool tone circle. The value of $\overrightarrow{\Delta i}$ of $\left(i_{x_{0}}, i_{y_{0}}\right)$ in warm tone area in Figure 11 indicates the minimum inclination maneuver of $\left(i_{x_{0}}, i_{y_{0}}\right)$ to keep $\left(i_{x}(t), i_{y}(t)\right)$ remained in the limited circle after 100 Earth days.

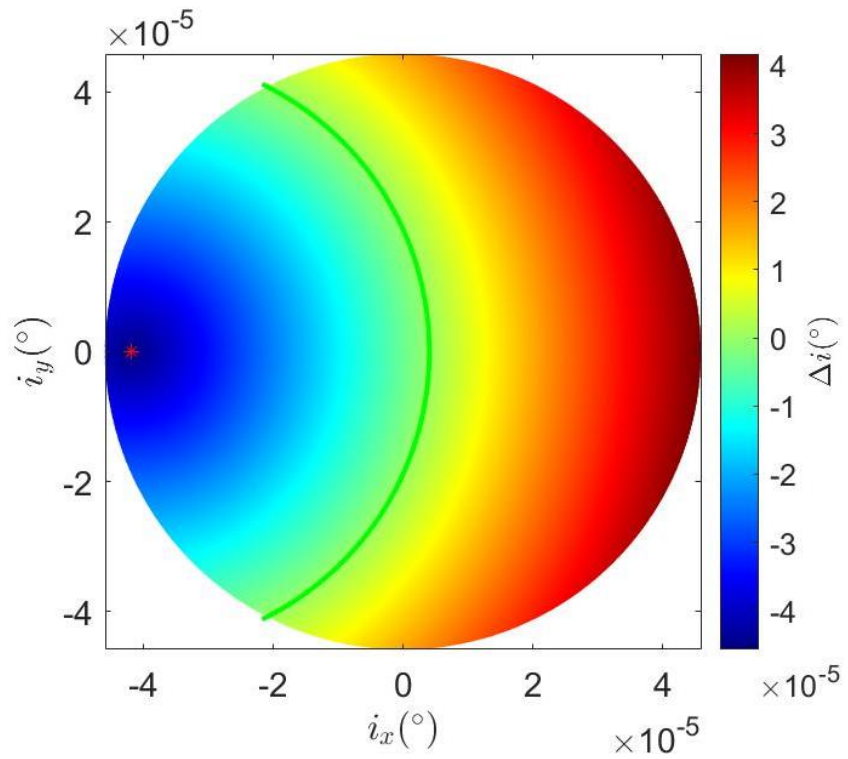

Figure 11. $\Delta i$ for control period $\Delta T_{C}=100$ Earth days with initial inclination vector point $\left(i_{x_{0}}, i_{y_{0}}\right)$ in the inclination limited circle whose radius $r=4.58 \times 10^{-5}$ deg perturbed by solar gravity.

After analyzing the effect caused by solar gravity perturbation, we now turn to the solar radiation pressure which may lead to eccentricity drift. First, the mean solar irradiance around Saturn is $P=15.04 \mathrm{~W} / \mathrm{m}^{2}$ while the distance from the Sun to Saturn is $1.426 \times 10^{12} \mathrm{~m}$ [38]. Then we find the intensity of solar radiation pressure is $p=\frac{P}{c}=$ $5.0168 \times 10^{-8} \mathrm{~N} / \mathrm{m}^{2}$, where $c=299,792,458 \mathrm{~m} / \mathrm{s}$ is the speed of light. Using other supposed parameters: the reflection parameter $K=1$, area of spacecraft vertical to the Sun $A=20 \mathrm{~m}^{2}$, the mass of spacecraft $m=3000 \mathrm{~kg}$, then the solar radiation pressure of spacecraft on stationary orbits around Saturn is [34]:

$$
\mathbf{F}_{\mathbf{s}}=-K p\left(\frac{A}{m}\right) \mathbf{S}=3.3445 \times 10^{-10} \mathbf{S}
$$

where $\mathbf{S}$ is the unit vector from the centroid of Saturn pointing at the Sun.

Using the Lagrange perturbation equations, we could find the average time derivatives of the eccentricity vector $e_{x}=e \cos (\omega+\Omega), e_{y}=e \sin (\omega+\Omega)$ in Equation (35).

$$
\begin{aligned}
\frac{d e_{x}}{d t} & =\frac{1}{2 \pi} \int_{0}^{2 \pi} \frac{1}{n a}\left(F_{r} \sin l+2 F_{t} \cos l\right) d l \\
& =-\frac{3 F_{s}}{2 n a} \sin l_{s} \cos i_{s}, \\
\frac{d e_{y}}{d t} & =\frac{1}{2 \pi} \int_{0}^{2 \pi} \frac{1}{n a}\left(-F_{r} \cos l+2 F_{t} \sin l\right) d l \\
& =\frac{3 F_{s}}{2 n a} \cos l_{s},
\end{aligned}
$$




$$
\begin{aligned}
& F_{r}=-\frac{F_{s}}{2}\left[\left(1-\cos i_{s}\right) \cos \left(\Omega+\omega+f+l_{s}\right)+\left(1+\cos i_{s}\right) \cos \left(\Omega+\omega+f-l_{s}\right)\right], \\
& F_{t}=\frac{F_{s}}{2}\left[\left(1-\cos i_{s}\right) \sin \left(\Omega+\omega+f+l_{s}\right)+\left(1+\cos i_{s}\right) \sin \left(\Omega+\omega+f-l_{s}\right)\right] .
\end{aligned}
$$

where $F_{r}$ and $F_{t}$ are the radial component and tangential component of solar radiation pressure $\mathbf{F}_{\mathbf{s}}$.

When we analyze the eccentricity perturbation of spacecraft in stationary orbits over a long time period, we learn that the motion of the Sun in a time period could be described as $\Delta l_{s}=l_{s}(t)-l_{s}\left(t_{0}\right)=n_{s}\left(t-t_{0}\right)$. Then we find the time integral of the eccentricity vector for any given $t$.

$$
\begin{aligned}
& e_{x}(t)=e_{x}\left(t_{0}\right)+\frac{3 F_{s}}{2 n a} \frac{1}{n_{s}}\left[\cos l_{s}(t)-\cos l_{s}\left(t_{0}\right)\right] \cos i_{s}, \\
& e_{y}(t)=e_{y}\left(t_{0}\right)+\frac{3 F_{s}}{2 n a} \frac{1}{n_{s}}\left[\sin l_{s}(t)-\sin l_{s}\left(t_{0}\right)\right] .
\end{aligned}
$$

We learn from Equation (37) that the curve of the eccentricity vector is an ellipse due to the existence of $\cos i_{s}$, and initial condition $\left(e_{x_{0}}, e_{y_{0}}\right)$ and $l_{s_{0}}$ influence the position of this eccentricity ellipse. From Figure 12, we learn that $l_{s_{0}}$, which means the angular between initial solar radiation direction and $x$ axis, can influence the perturbing direction. The centre of eccentricity limitation circle is $\left(e_{x_{0}}, e_{y_{0}}\right)$.

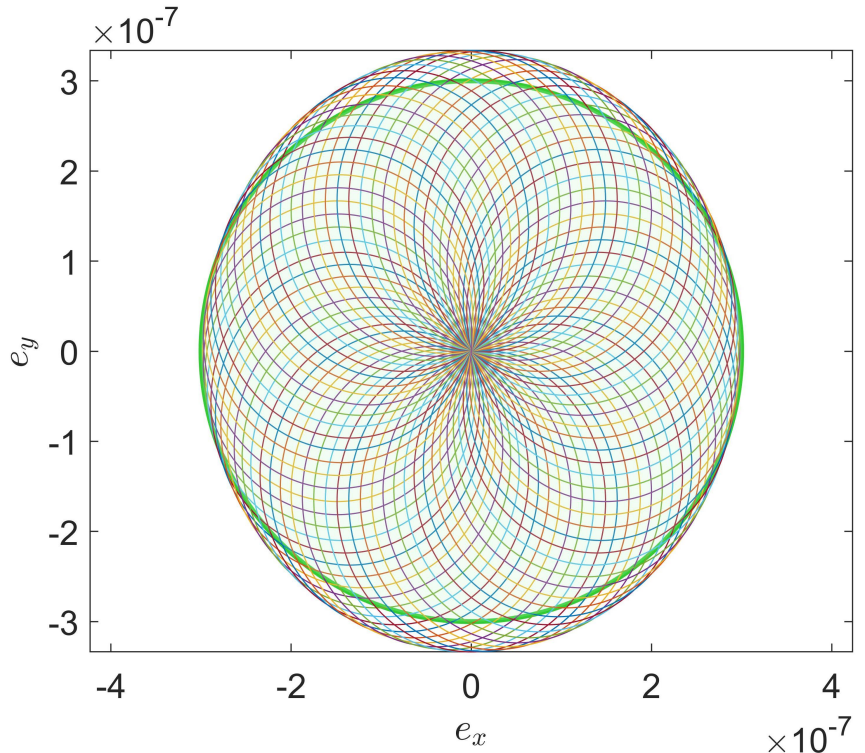

Figure 12. A total of 72 curves of $f\left(e_{x}, e_{y}\right)$ perturbed by solar radiation pressure for $l_{s 0}=5 n \mathrm{deg}$, the integral $n \in[0,72]$, in a Saturnian year with initial eccentricity vector $\left(e_{x_{0}}, e_{y_{0}}\right)=(0,0)$ in the eccentricity perturbation limited circle whose radius $r=3 \times 10^{-7}$.

Here, we fix the $\left(e_{x_{0}}, e_{y_{0}}\right)=(0,0)$. When $\left(e_{x_{0}}, e_{y_{0}}\right) \neq(0,0)$, we can first take a maneuver to make the point $\left(e_{x_{0}}, e_{y_{0}}\right)=(0,0)$ in the curve of eccentricity perturbation. Then, we can design a maintenance strategy. When taking an eccentricity control, we used to set $\Delta V_{t}>0$, then we could suppose $B=-\frac{\Delta V_{t}}{T}$, and find the maneuver function [39]:

$$
\begin{aligned}
& \frac{d e_{x}}{d t}=-\frac{2}{n a} B \cos \left(l_{s}-\frac{\pi}{2}\right), \\
& \frac{d e_{y}}{d t}=-\frac{2}{n a} B \sin \left(l_{s}-\frac{\pi}{2}\right) .
\end{aligned}
$$

Combined with solar radiation pressure perturbation Equation (35), the rate of eccentricity vector $\left(e_{x}, e_{y}\right)$ after a maneuver could be rewritten as 


$$
\begin{aligned}
\frac{d e_{x}}{d t} & =-\frac{3 F_{s}}{2 n a} \sin l_{s} \cos i_{s}+\frac{2}{n a} B \cos \left(l_{s}-\frac{\pi}{2}\right) \\
& =-\frac{3 F_{s}}{2 n a} \sin l_{s} \cos i_{s}-\frac{2}{n a} B \sin l_{s}, \\
\frac{d e_{y}}{d t} & =\frac{3 F_{s}}{2 n a} \cos l_{s}+\frac{2}{n a} B \sin \left(l_{s}-\frac{\pi}{2}\right) \\
& =\frac{3 F_{s}}{2 n a} \cos l_{s}+\frac{2}{n a} B \cos l_{s} .
\end{aligned}
$$

We still integrate Equation (38), then we could find the eccentricity vector at any time $t$ after we take an eccentricity control maneuver.

$$
\begin{aligned}
& e_{x}(t)=e_{x}\left(t_{0}\right)+\frac{3 F_{s}}{2 n a} \frac{1}{n_{s}}\left[\cos l_{s}(t)-\cos l_{s}\left(t_{0}\right)\right] \cos i_{s}+\frac{2}{n a} \frac{B}{n_{s}}\left[\cos l_{s}(t)-\cos l_{s}\left(t_{0}\right)\right], \\
& e_{y}(t)=e_{y}\left(t_{0}\right)+\frac{3 F_{s}}{2 n a} \frac{1}{n_{s}}\left[\sin l_{s}(t)-\sin l_{s}\left(t_{0}\right)\right]+\frac{2}{n a} \frac{B}{n_{s}}\left[\sin l_{s}(t)-\sin l_{s}\left(t_{0}\right)\right] .
\end{aligned}
$$

To find a better control effect, we take 6 times eccentricity control for different initial solar radiation direction.

$$
\begin{aligned}
& e_{x}(t)=e_{x}\left(t_{0}\right)+\frac{3 F_{s}}{2 n a} \frac{1}{n_{s}}\left[\cos l_{s}(t)-\cos l_{s}\left(t_{0}\right)\right] \cos i_{s}+\sum_{i=1}^{6} \frac{2}{n a} \frac{B}{n_{s}}\left[\cos l_{s}\left(t_{i}\right)-\cos l_{s}\left(t_{i_{0}}\right)\right], \\
& e_{y}(t)=e_{y}\left(t_{0}\right)+\frac{3 F_{s}}{2 n a} \frac{1}{n_{s}}\left[\sin l_{s}(t)-\sin l_{s}\left(t_{0}\right)\right]+\sum_{i=1}^{6} \frac{2}{n a} \frac{B}{n_{s}}\left[\sin l_{s}\left(t_{i}\right)-\sin l_{s}\left(t_{i_{0}}\right)\right],
\end{aligned}
$$

where

$$
\begin{aligned}
& B=-5 \times 10^{-11} \mathrm{~m} / \mathrm{s}^{2} \\
& l_{s}\left(t_{0}\right)=0 \mathrm{deg}, \\
& l_{s}\left(t_{i_{0}}\right)=60 \mathrm{deg} \times i, i=1 \ldots 6 .
\end{aligned}
$$

Compared Figure 12 with Figure 13, we could know that $e_{x}, e_{y}$ are kept staying in the eccentricity limited circle after a Saturn's year for initial $\left(e_{x_{0}}, e_{y_{0}}\right)=(0,0)$ and any given solar radiation direction $l_{s_{0}}$.

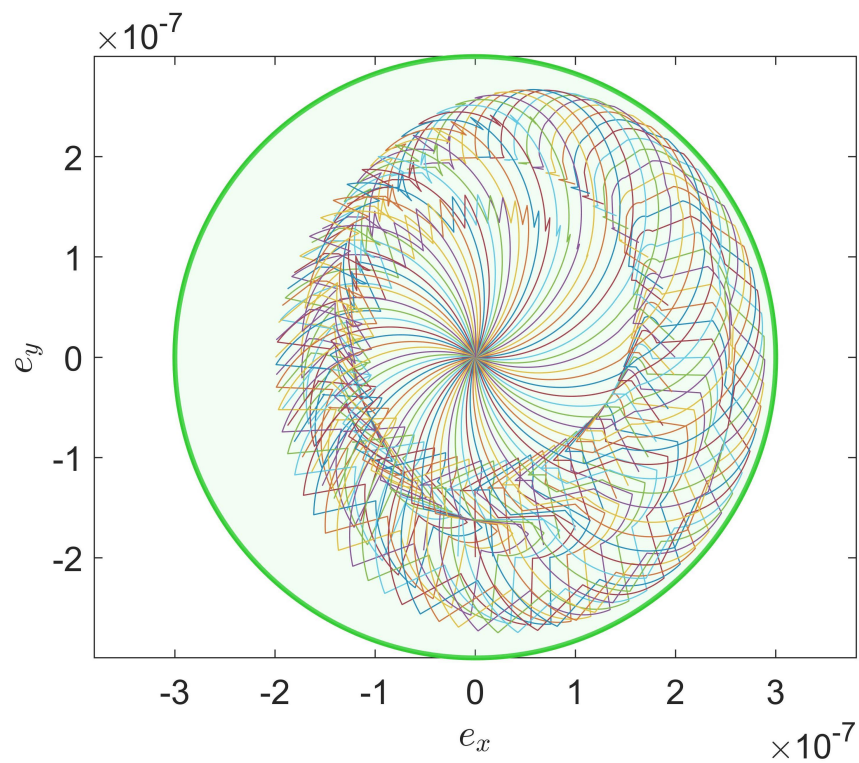

Figure 13. After taking 6 times eccentricity control when $B=-5 \times 10^{-11} \mathrm{~m} / \mathrm{s}^{2}, 72$ curves of $f\left(e_{x}, e_{y}\right)$ perturbed by solar radiation pressure for $l_{s_{0}}=5 n \mathrm{deg}$, integral $n \in[0,72]$ in a Saturnian year with initial eccentricity vector point $\left(e_{x_{0}}, e_{y_{0}}\right)=(0,0)$ in the eccentricity perturbation limited circle whose radius $r=3 \times 10^{-7}$. 


\section{Conclusions}

In our paper, we analyze the sun-synchronous orbits, repeating ground track orbits, frozen orbits, and stationary orbits around Saturn and corresponding control strategies based on the mean element theory while zonal harmonic coefficients $J_{2}$ and $J_{4}$ of Saturnian gravitational field are considered.

For sun-synchronous orbits, we analyze the existence of orbits, find the relation between inclination with eccentricity and semi major axis, and calculate the local time drift caused by solar gravitation and atmospheric drag. After that, we take two inclinationbiased control strategies to damp the local time drift. The initial inclination-biased method suits the short-time spacecraft. The periodic inclination biased method has a better effect on long-period missions.

For repeating ground track orbits, we find the meaningful range of repetition parameter $Q$. Then we find the relation between the inclination and semimajor axis. After that, we calculate the compensation for semimajor axis and maneuver period.

For frozen orbits, we learn that only when $\omega=270 \mathrm{deg}$ can we be sure of the eccentricity positive for any given inclination and semimajor axis.

For stationary orbits, we first calculate the radius using the conditions of equilibrium point. Then, we analyze the perturbations caused by solar gravitation and solar radiation pressure. Finally, we take corresponding maneuver strategies to control the inclination and eccentricity.

Author Contributions: Conceptualization, C.Z. and Y.L.; methodology, C.Z. and Y.J.; software, C.Z.; validation, C.Z. and Y.J.; formal analysis, H.L.; resources, Y.J.; data curation, C.Z. and Y.L.; writing - original draft preparation, data curation, and writing-review and editing, H.L. and Y.J.; visualization, C.Z. and Y.L.; supervision, H.L. and Y.J.; project administration, H.L. and Y.J.; and funding acquisition, Y.J. All authors have read and agreed to the published version of the manuscript.

Funding: This research was funded by the National Natural Science Foundation of China (Grant No. 11772356, No. U21B2050).

Institutional Review Board Statement: Not applicable.

Informed Consent Statement: Not applicable.

Acknowledgments: The authors gratefully acknowledge the reviewers for their careful work and thoughtful suggestions that have helped improve this paper substantially.

Conflicts of Interest: The authors declare no conflict of interest.

\section{References}

1. Available online: https://nssdc.gsfc.nasa.gov/planetary/factsheet/saturnfact.html (accessed on 10 December 2021).

2. Available online: https://nssdc.gsfc.nasa.gov/planetary/factsheet/saturniansatfact.html (accessed on 10 December 2021).

3. Available online: https://solarsystem.nasa.gov/missions/pioneer-11/in-depth/ (accessed on 10 December 2021).

4. Available online: https://solarsystem.nasa.gov/missions/voyager-2/in-depth/ (accessed on 10 December 2021).

5. Available online: https://solarsystem.nasa.gov/missions/cassini/overview/ (accessed on 10 December 2021).

6. Jacobson, R.A. The orbits of the major Saturnian satellites and the gravity field of saturn from spacecraft and earth-based observations. Astron. J. 2004, 128, 492-501. [CrossRef]

7. Jacobson, R.A.; Antreasian, P.G.; Bordi, J.J.; Criddle, K.E.; Ionasescu, R.; Jones, J.B.; Mackenzie, R.A.; Meek, M.C.; Parcher, D.; Pelletier, F.J.; et al. The gravity field of the Saturnian system from satellite observations and spacecraft tracking data. Astron. J. 2006, 132, 2520-2526. [CrossRef]

8. Waite, J.H., Jr.; Perryman, R.S.; Perry, M.E.; Miller, K.E.; Bell, J.; Cravens, T.E.; Glein, C.R.; Grimes, J.; Hedman, M.; Cuzzi, J.; et al. Chemical interactions between Saturn's atmosphere and its rings. Science 2018, 362, 51. [CrossRef] [PubMed]

9. Müller-Wodarg, I.C.F.; Moore, L.; Galand, M.; Miller, S.; Mendillo, M. Magnetosphere-atmosphere coupling at Saturn: 1Response of thermosphere and ionosphere to steady state polar forcing. Icarus 2012, 221, 481-494. [CrossRef]

10. Koskinen, T.T.; Guerlet, S. The Atmospheric structure and helium abundance on Saturn from Cassini/UVIS and CIRS observations. Icarus 2018, 307, 161-171. [CrossRef]

11. Badman, S.V.; Jackman, C.M.; Nichols, J.D.; Clarke, J.T.; Gérard, J.C. Open flux in Saturn's magnetosphere. Icarus 2014, 231, 137-145. [CrossRef] 
12. Tiscareno, M.S.; Nicholson, P.D.; Cuzzi, J.N.; Spilker, L.J.; Murray, C.D.; Hedman, M.M.; Colwell, J.E.; Burns, J.A.; Brooks, S.M.; Clark, R.N.; et al. Close-range remote sensing of Saturn's rings during Cassini's ring-grazing orbits and Grand Finale. Science 2019, 364, eaau1017. [CrossRef]

13. Iess, L.; Rappaport, N.J.; Jacobson, R.A.; Racioppa, P.; Stevenson, D.J.; Tortora, P.; Armstrong, J.W.; Asmar, S.W. Gravity field, shape and moment of inertia of Titan. Science 2010, 327, 1367-1369. [CrossRef]

14. Spilker, L. Cassini-Huygens' exploration of the Saturn system: 13 years of discovery. Science 2019, 364, 1046-1051. [CrossRef]

15. Kozai, Y. The motion of a close earth satellite. Astron. J. 1959, 64, 367. [CrossRef]

16. Brouwer, D. Solution of the problem of artificial satellite theory without drag. Astron. J. 1959, 64, 378. [CrossRef]

17. Cutting, E.; Born, G.H.; Frautnick, J.C. Orbit analysis for Seasat-A. J. Astronaut. Sci. 1978, 26, 315-342.

18. Musen, P.; Bailie, A.E. On the motion of a 24-hour satellite. J. Geophys. Res. 1962, 67, 1123-1132. [CrossRef]

19. Lara, M.; Elipe, A. Periodic orbits around geostationary positions. Celest. Mech. Dyn. Astron. 2002, 82, 285-299. [CrossRef]

20. Lei, H. Dynamical models for secular evolution of navigation satellites. Astrodynamics 2019, 4, 57-73. [CrossRef]

21. Nazarenko, A.I. Sun synchronous orbits. Predicting the local solar time of the ascending node. Acta Astronaut. 2021, 181, 585-593. [CrossRef]

22. Liao, C.; Xu, M.; Jia, X.; Dong, Y. Semi-analytical acquisition algorithm for repeat-groundtrack orbit maintenance. Astrodynamics 2018, 2, 161-173. [CrossRef]

23. Liu, X.; Baoyin, H.; Ma, X. Five Special Types of Orbits Around Mars. J. Guid. Control Dyn. 2010, 33, 1294-1301. [CrossRef]

24. Liu, X.; Baoyin, H.; Ma, X. Periodic orbits around areostationary points in the Martian gravity field. Res. Astron. Astrophys. 2012, 12, 551-562. [CrossRef]

25. Ortore, E.; Circi, C.; Ulivieri, C.; Cinelli, M. Multi-SunSynchronous Orbits in the Solar System. Earth Moon Planets 2014, $111,157$. [CrossRef]

26. Ma, X.; Li, J. Distant quasi-periodic orbits around Mercury. Astrophys. Space Sci. 2013, 343, 83-93. [CrossRef]

27. Ma, X.; Li, J. Artificial frozen orbits around Mercury. Astrophys. Space Sci. 2013, 348, 345-365. [CrossRef]

28. Liu, Y.; Jiang, Y.; Li, H.; Zhang, H. Some Special Types of Orbits around Jupiter. Aerospace 2021, 8, 183. [CrossRef]

29. Jiang, C.; Liu, Y.; Jiang, Y.; Li, H. Orbital Design and Control for Jupiter-Observation Spacecraft. Aerospace 2021, 8, 282. [CrossRef]

30. Galanti, E.; Kaspi, Y. Combined magnetic and gravity measurements probe the deep zonal flows of the gas giants. Mon. Not. R. Astron. Soc. 2021, 501, 2352-2362. [CrossRef]

31. Roy, A.E. Orbital Motion, 4th ed.; Taylor \& Francis Group: New York, NY, USA, 2005; pp. 205-206.

32. Liu, L. Orbit Theory of Spacecraft; National Defense Industry Press: Beijing, China, 2000. (In Chinese)

33. Jiang, Y.; Baoyin, H.; Zhang, Y. Relative effect of inclinations for moonlets in the triple asteroidal systems. Earth Moon Planets 2017, 64, 133-137. [CrossRef]

34. Cook, G.E. Luni-Solar perturbations of the orbit of an earth satellite. Geophys. J. R. Astron. Soc. 1962, 6, 271-291. [CrossRef]

35. Capderou, M. Handbook of Satellite Orbits: From Kepler to GPS, Perturbative Method Results for the Geopotential up to J ${ }_{2}$; Springer International Publishing: Cham, Switzwerland, 2014; pp. 191-201.

36. Capderou, M. Handbook of Satellite Orbits: From Kepler to GPS, Appendix: Atmospheric Drag; Springer International Publishing: Cham, Switzwerland, 2014; pp. 204-210.

37. Liu, J.J.F. Satellite Motion about an Oblate Earth. AIAA J. 1974, 12, 1511-1516. [CrossRef]

38. Available online: https://www.pveducation.org/pvcdrom/properties-of-sunlight/solar-radiation-in-space (accessed on 10 December 2021).

39. Xie, Y.; Lei, Y.; Guo, J.; Meng, B. Spacecraft Dynamics and Control; Springer Nature Singapore PTE Ltd.: Singapore, $2022 ;$ p. 108 